\title{
Probe of New Physics using Precision Measurement of the Electron Magnetic Moment
}

\author{
Amin Aboubrahim ${ }^{41}$, Tarek Ibrahim ${ }^{a, 42}$, and Pran Nath ${ }^{3}$ \\ ${ }^{a}$ Department of Physics, Faculty of Science, University of Alexandria, Alexandria 21511, Egypt \\ ${ }^{b}$ Department of Physics, Faculty of Sciences, Beirut Arab University, Beirut $11-5020$, Lebanon ${ }^{5}$ \\ ${ }^{c}$ Department of Physics, Northeastern University, Boston, Massachusetts 02115-5000, USA
}

\begin{abstract}
The anomalous magnetic moment of the electron is determined experimentally with an accuracy of $2.8 \times 10^{-13}$ and the uncertainty may decrease by an order of magnitude in the future. While the current data is in excellent agreement with the standard model, the possible future improvement in the error in $\Delta a_{e}=a_{e}^{\text {exp }}-a_{e}^{\text {theory }}$ has recently drawn interest in the electron anomalous magnetic moment as a possible probe of new physics beyond the standard model. In this work we give an analysis of such physics in an extension of the minimal supersymmetric standard model with a vector multiplet. In the extended model the electroweak contribution to the anomalous magnetic moment of the electron include loop diagrams involving in addition to the exchange of $\mathrm{W}$ and $\mathrm{Z}$, the exchange of charginos, sneutrinos and mirror sneutrinos, and the exchange of neutralinos, sleptons and mirror sleptons. The analysis shows that a contribution to the electron magnetic moment much larger than expected by $m_{e}^{2} / m_{\mu}^{2}$ scaling of the deviation of the muon anomalous magnetic moment over the standard model prediction, i.e., $\Delta a_{\mu}=3 \times 10^{-9}$ as given by the Brookhaven experiment, can be gotten within the MSSM extension. Effects of CP violating phases in the extended MSSM model on the corrections to the supersymmetric electroweak contributions to $a_{e}$ are also investigated. The analysis points to the possibility of detection of new physics effects with modest improvement on the error in $\Delta a_{e}=a_{e}^{\text {exp }}-a_{e}^{\text {theory }}$.
\end{abstract}

Keywords: Anomalous magnetic dipole moments, supersymmetry, vector lepton multiplets

PACS numbers: 13.40Em, 12.60.-i, 14.60.Fg

\footnotetext{
${ }^{1}$ Email: amin.b@bau.edu.lb

${ }^{2}$ Email: t.ibrahim@bau.edu.lb

${ }^{3}$ Emal: nath@neu.edu

${ }^{4}$ Permanent address.

${ }^{5}$ Current address.
} 


\section{Introduction}

The anomalous magnetic moment of the electron $a_{e}=(g-2) / 2$ is one of most accurately determined quantities experimentally. Thus the most recent determination of it gives the value [1]

$$
a_{e}^{\exp }=11596521807.3(2.8) \times 10^{-13}
$$

In the standard model the contribution to the magnetic moment of the electron arises from several sources so that (for a review see [2])

$$
a_{e}^{\mathrm{SM}}=a_{e}^{\mathrm{qed}}+a_{e}^{\mathrm{EW}}+a_{e}^{\mathrm{had}}
$$

where $a_{e}^{\text {qed }}$ involves purely QED corrections and includes one loop[3], two loop [4], three loop [5], 4 loop [6, 7] and more recently five loop [8, 9, 10] contributions. Specifically in this work we will be using the results of [9] which is an impressive work giving the complete up to tenth -order QED contribution to $(g-2)_{e}$. The analysis of [9] also improves the eighth-order contribution which includes the mass-dependent contributions. (In the context of precision analyses of $(g-2)_{e}$ and their sensitivity to higher terms in the ratio of the masses see also [7, 11]). $a_{e}^{\mathrm{EW}}[12$ contains the electroweak corrections involving the $W$ and $Z$ loops, and $a_{e}^{\text {had }}$ contains the hadronic corrections [13, 14. Now in comparing the theory prediction with experiment one must use in the computation of the theory prediction the value of $\alpha$ obtained in independent experiment rather than by equating $a_{e}^{\mathrm{SM}}(\alpha)=a_{e}^{\exp }$ [9, 15]. Thus the analysis of [9] uses the value of $\alpha$ obtained from the measurement of $h / m_{\mathrm{Rb}}$ [16] combined with the accurately known Rydberg constant and $m_{\mathrm{Rb}} / m_{e}$ (for a review see [17]) which gives

$$
\alpha^{-1}\left({ }^{87} \mathrm{Rb}\right)=1 / 137.035999049(90)
$$

Using Eq.(3) the analysis of [9] gives

$$
a_{e}^{\mathrm{SM}}=1159652181.78(6)(4)(3)(77) \times 10^{-12},
$$

where the numbers in the parentheses are as follows: (6) refers to the uncertainty in the four loop QED coefficient, (4) refers to the uncertainty in the five loop QED co-efficient, (3) is the error in the hadronic contribution, and (77) arises from the error in the determination of $\alpha$ using ${ }^{87} \mathrm{Rb}$ data. Combining the errors in quadratures one finds [9] that the uncertainty $\delta \Delta a_{e}$, where $\Delta a_{e}=\left(a_{e}^{\exp }-a_{e}^{\mathrm{SM}}\right)$, is given by

$$
\delta \Delta a_{e}=8.2 \times 10^{-13}
$$


Similar to the work of [15] our motivation is to use Eq. (5) to constrain new physics. Specifically we look now at the implications of Eq.(5) in view of the current status of the anomalous magnetic moment of the muon. Thus the Brookhaven experiment indicates a $\sim 3.5 \sigma$ deviation from the standard model prediction, i.e., one has for $\Delta a_{\mu}$ the result [18, 19]

$$
\Delta a_{\mu}=(287 \pm 80) \times 10^{-11}
$$

Scaling the result of Eq. (6) to the case of the electron by using the naive scaling factor of $m_{e}^{2} / m_{\mu}^{2}$ one gets a correction of size $(0.6 \pm 0.2) \times 10^{-13}$ which is an order of magnitude smaller than the result of Eq.(5). The above discussion indicates that if there are new physics effects larger than those given by naive scaling, they would be susceptible to discovery with modest improvements in the error $\delta \Delta a_{e}$.

In this work we carry out a detailed analysis of corrections to the anomalous magnetic moment of the electron in extensions of MSSM with a vector multiplet (For a non-supersymmetric analysis see also [20, 15]). The analysis will include contributions from the $\mathrm{W}$ and $\mathrm{Z}$ boson loops, as well as corrections from charginos, sneutrinos and mirror sneutrinos, from neutralinos and sleptons and mirror sleptons. It will be shown that the new physics corrections here can be far in excess of those implied by scaling and are of a size that could be detectable in modest improvement in $\delta \Delta a_{e}$. We also investigate the dependence of the anomalous magnetic moment of the electron on CP phases arising from the supersymmetric contributions from the exchange of the vectorlike multiplet. In previous analyses within MSSM the supersymmetric correction to the anomalous magnetic moment of the muon was found to be sensitive to $\mathrm{CP}$ phases in a significant way [22] and we could have similar large $\mathrm{CP}$ dependent effects for $\Delta a_{e}(\mathrm{EW})$ in the analysis based on the MSSM extension.

The outline of the rest of the paper is as follows: In Section 2 we discuss the MSSM extension with a vectorlike multiplet. Here we define the notation labeling the extra vectorlike particles, give their transformation properties under the SM gauge group and give the superpotential for the extended model. The D terms and the soft terms allowed in the model are discussed. In Section 3 the interactions of leptons-sneutrinos (mirror sneutrinos)-charginos in the mass diagonal basis are given. These interactions are used in the computation of the left diagram of Fig. 1. In Section 4 the interactions of leptons-sleptons (mirror sleptons)-neutralinos in the mass diagonal basis are given. These interactions are used in the computation of the right diagram of Fig. 1. In Section 5 the interactions of the $\mathrm{W}$ and $\mathrm{Z}$ bosons that are needed in the computation of the 
loop diagram of Fig. 2 are discussed. In Section 6 an analytic analysis is given of the neutralino exchange contributions using the interactions of Section 3 and chargino exchange contribution using the interaction of Section 4. Here an analytic analysis is also given of the exchange contributions of the $\mathrm{W}$ and $\mathrm{Z}$ bosons using the interactions of Fig. 2. A detailed numerical analysis is given in Section 7 for the electroweak contribution to the electron anomalous magnetic moment in the model. Here the dependence of electroweak contribution to the anomalous magnetic moment of the electron on supersymmetric CP phases is also investigated. It is shown that modest improvements in the current errors in $\Delta a_{e}$ can begin to probe the possible new physics contributions. Further, a relative comparison of the electroweak contributions to the anomalous magnetic moments of $e, \mu, \tau$ is also given. Conclusions are given in Section 8. Further details on the mass matrices for the sleptons and mirror sleptons are given in Section 9 .

\section{MSSM Extension with a vector leptonic multiplet}

Vector like multiplets arise in a variety of unified models [23] some of which could be low lying. They have been used recently in a variety of analyses [24, 25, 26, 27, 28, 29, 30, 31, 32, 33, 34. In the analysis below we will assume an extended MSSM with just one leptonic vector mulitplet. The addition of a vector multiplet keeps the model anomaly free. Before proceeding further we define the notation and give a very brief description of the extended model and a more detailed description can be found in the previous works mentioned above. Thus the extended MSSM has contains a vectorlike multiplet with the transformations under $S U(3)_{C} \times S U(2)_{L} \times U(1)_{Y}$ as given below

$$
\begin{array}{rrr}
\psi_{i L} \equiv\left(\begin{array}{c}
\nu_{i L} \\
l_{i L}
\end{array}\right) & l_{i L}^{c} & \nu_{i L}^{c} \\
\left(1,2,-\frac{1}{2}\right) & (1,1,1) & (1,1,0)
\end{array}
$$

where the last entry on the right hand side column is the value of the hypercharge $Y$ defined so that $Q=T_{3}+Y$. These leptons have $V-A$ interactions. We can now add a vectorlike multiplet where we have a fourth family of leptons with $V-A$ interactions whose transformations can be gotten from Eq. (7) by letting i run from 1-4. A vectorlike lepton multiplet also has mirrors and so we consider these mirror leptons which have $V+A$ interactions. Its quantum numbers are given by 


$$
\begin{array}{rrr}
\chi^{c} \equiv\left(\begin{array}{c}
E_{L}^{c} \\
N_{L}^{c}
\end{array}\right) & E_{L} & N_{L} \\
\left(1,2, \frac{1}{2}\right) & (1,1,-1) & (1,1,0) .
\end{array}
$$

Interesting new physics arises when we allow mixings of the vectorlike generation with the three ordinary generations. Thus the superpotential of the model allowing for the mixings among the three ordinary generations and the vectorlike generation is given by

$$
\begin{aligned}
W & =-\mu \epsilon_{i j} \hat{H}_{1}^{i} \hat{H}_{2}^{j}+\epsilon_{i j}\left[f_{1} \hat{H}_{1}^{i} \hat{\psi}_{L}^{j} \hat{\tau}_{L}^{c}+f_{1}^{\prime} \hat{H}_{2}^{j} \hat{\psi}_{L}^{i} \hat{\nu}_{\tau L}^{c}+f_{2} \hat{H}_{1}^{i} \hat{\chi}^{c j} \hat{N}_{L}+f_{2}^{\prime} H_{2}^{j} \hat{\chi}^{c i} \hat{E}_{L}\right. \\
& \left.+h_{1} H_{1}^{i} \hat{\psi}_{\mu L}^{j} \hat{\mu}_{L}^{c}+h_{1}^{\prime} H_{2}^{j} \hat{\psi}_{\mu L}^{i} \hat{\nu}_{\mu L}^{c}+h_{2} H_{1}^{i} \hat{\psi}_{e L}^{j} \hat{e}_{L}^{c}+h_{2}^{\prime} H_{2}^{j} \hat{\psi}_{e L}^{i} \hat{\nu}_{e L}^{c}\right] \\
& +f_{3} \epsilon_{i j} \hat{\chi}^{c i} \hat{\psi}_{L}^{j}+f_{3}^{\prime} \epsilon_{i j} \hat{\chi}^{c i} \hat{\psi}_{\mu L}^{j}+f_{4} \hat{\tau}_{L}^{c} \hat{E}_{L}+f_{5} \hat{\nu}_{\tau L}^{c} \hat{N}_{L}+f_{4}^{\prime} \hat{\mu}_{L}^{c} \hat{E}_{L}+f_{5}^{\prime} \hat{\nu}_{\mu L}^{c} \hat{N}_{L} \\
& +f_{3}^{\prime \prime} \epsilon_{i j} \hat{\chi}^{c i} \hat{\psi}_{e L}^{j}+f_{4}^{\prime \prime} \hat{e}_{L}^{c} \hat{E}_{L}+f_{5}^{\prime \prime} \hat{\nu}_{e L}^{c} \hat{N}_{L},
\end{aligned}
$$

where ${ }^{\wedge}$ implies superfields, $\hat{\psi}_{L}$ stands for $\hat{\psi}_{3 L}, \hat{\psi}_{\mu L}$ stands for $\hat{\psi}_{2 L}$ and $\hat{\psi}_{e L}$ stands for $\hat{\psi}_{1 L}$. The mass terms for the neutrinos, mirror neutrinos, leptons and mirror leptons arise from the term

$$
\mathcal{L}=-\frac{1}{2} \frac{\partial^{2} W}{\partial A_{i} \partial A_{j}} \psi_{i} \psi_{j}+\text { H.c. }
$$

where $\psi$ and $A$ stand for generic two-component fermion and scalar fields. After spontaneous breaking of the electroweak symmetry, $\left(\left\langle H_{1}^{1}\right\rangle=v_{1} / \sqrt{2}\right.$ and $\left.\left\langle H_{2}^{2}\right\rangle=v_{2} / \sqrt{2}\right)$, we have the following set of mass terms written in the 4-component spinor notation so that

$$
-\mathcal{L}_{m}=\bar{\xi}_{R}^{T}\left(M_{f}\right) \xi_{L}+\bar{\eta}_{R}^{T}\left(M_{\ell}\right) \eta_{L}+\text { H.c. }
$$

where the basis vectors in which the mass matrix is written is given by

$$
\begin{gathered}
\bar{\xi}_{R}^{T}=\left(\begin{array}{llll}
\bar{\nu}_{\tau R} & \bar{N}_{R} & \bar{\nu}_{\mu R} & \bar{\nu}_{e R}
\end{array}\right), \\
\xi_{L}^{T}=\left(\begin{array}{llll}
\nu_{\tau L} & N_{L} & \nu_{\mu L} & \nu_{e L}
\end{array}\right), \\
\bar{\eta}_{R}^{T}=\left(\begin{array}{llll}
\overline{\tau_{R}} & \bar{E}_{R} & \overline{\mu_{R}} & \overline{e_{R}}
\end{array}\right), \\
\eta_{L}^{T}=\left(\begin{array}{llll}
\tau_{L} & E_{L} & \mu_{L} & e_{L}
\end{array}\right),
\end{gathered}
$$

and the mass matrix $M_{f}$ is given by

$$
M_{f}=\left(\begin{array}{cccc}
f_{1}^{\prime} v_{2} / \sqrt{2} & f_{5} & 0 & 0 \\
-f_{3} & f_{2} v_{1} / \sqrt{2} & -f_{3}^{\prime} & -f_{3}^{\prime \prime} \\
0 & f_{5}^{\prime} & h_{1}^{\prime} v_{2} / \sqrt{2} & 0 \\
0 & f_{5}^{\prime \prime} & 0 & h_{2}^{\prime} v_{2} / \sqrt{2}
\end{array}\right)
$$


We define the matrix element (22) of the mass matrix as $m_{N}$ so that

$$
m_{N}=f_{2} v_{1} / \sqrt{2}
$$

The mass matrix is not hermitian and thus one needs bi-unitary transformations to diagonalize it. We define the bi-unitary transformation so that

$$
D_{R}^{\nu \dagger}\left(M_{f}\right) D_{L}^{\nu}=\operatorname{diag}\left(m_{\psi_{1}}, m_{\psi_{2}}, m_{\psi_{3}}, m_{\psi_{4}}\right) .
$$

Under the bi-unitary transformations the basis vectors transform so that

$$
\left(\begin{array}{c}
\nu_{\tau_{R}} \\
N_{R} \\
\nu_{\mu_{R}} \\
\nu_{e_{R}}
\end{array}\right)=D_{R}^{\nu}\left(\begin{array}{l}
\psi_{1_{R}} \\
\psi_{2_{R}} \\
\psi_{3_{R}} \\
\psi_{4_{R}}
\end{array}\right), \quad\left(\begin{array}{c}
\nu_{\tau_{L}} \\
N_{L} \\
\nu_{\mu_{L}} \\
\nu_{e_{L}}
\end{array}\right)=D_{L}^{\nu}\left(\begin{array}{l}
\psi_{1_{L}} \\
\psi_{2_{L}} \\
\psi_{3_{L}} \\
\psi_{4_{L}}
\end{array}\right)
$$

In Eq. (15) $\psi_{1}, \psi_{2}, \psi_{3}, \psi_{4}$ are the mass eigenstates for the neutrinos, where in the limit of no mixing we identify $\psi_{1}$ as the light tau neutrino, $\psi_{2}$ as the heavier mass eigen state, $\psi_{3}$ as the muon neutrino and $\psi_{4}$ as the electron neutrino. A similar analysis goes to the lepton mass matrix $M_{\ell}$ where

$$
M_{\ell}=\left(\begin{array}{cccc}
f_{1} v_{1} / \sqrt{2} & f_{4} & 0 & 0 \\
f_{3} & f_{2}^{\prime} v_{2} / \sqrt{2} & f_{3}^{\prime} & f_{3}^{\prime \prime} \\
0 & f_{4}^{\prime} & h_{1} v_{1} / \sqrt{2} & 0 \\
0 & f_{4}^{\prime \prime} & 0 & h_{2} v_{1} / \sqrt{2}
\end{array}\right) .
$$

We introduce now the mass parameter $m_{E}$ defined by the (22) element of the mass matrix above so that

$$
m_{E}=f_{2}^{\prime} v_{2} / \sqrt{2}
$$

Next we consider the mixing of the charged sleptons and the charged mirror sleptons. The mass squared matrix of the slepton - mirror slepton comes from three sources: the F term, the D term of the potential and the soft susy breaking terms. Using the superpotential of Eq. (9) the mass terms arising from it after the breaking of the electroweak symmetry are given by the Lagrangian

$$
\mathcal{L}=\mathcal{L}_{F}+\mathcal{L}_{D}+\mathcal{L}_{\text {soft }}
$$

where $\mathcal{L}_{F}$ is deduced from Eq. (9) and is given in the Appendix, while the $\mathcal{L}_{D}$ is given by

$$
\begin{aligned}
-\mathcal{L}_{D} & =\frac{1}{2} m_{Z}^{2} \cos ^{2} \theta_{W} \cos 2 \beta\left\{\tilde{\nu}_{\tau L} \tilde{\nu}_{\tau L}^{*}-\tilde{\tau}_{L} \tilde{\tau}_{L}^{*}+\tilde{\nu}_{\mu L} \tilde{\nu}_{\mu L}^{*}-\tilde{\mu}_{L} \tilde{\mu}_{L}^{*}+\tilde{\nu}_{e L} \tilde{\nu}_{e L}^{*}-\tilde{e}_{L} \tilde{e}_{L}^{*}\right. \\
& \left.+\tilde{E}_{R} \tilde{E}_{R}^{*}-\tilde{N}_{R} \tilde{N}_{R}^{*}\right\}+\frac{1}{2} m_{Z}^{2} \sin ^{2} \theta_{W} \cos 2 \beta\left\{\tilde{\nu}_{\tau L} \tilde{\nu}_{\tau L}^{*}+\tilde{\tau}_{L} \tilde{\tau}_{L}^{*}+\tilde{\nu}_{\mu L} \tilde{\nu}_{\mu L}^{*}+\tilde{\mu}_{L} \tilde{\mu}_{L}^{*}\right. \\
& \left.+\tilde{\nu}_{e L} \tilde{\nu}_{e L}^{*}+\tilde{e}_{L} \tilde{e}_{L}^{*}-\tilde{E}_{R} \tilde{E}_{R}^{*}-\tilde{N}_{R} \tilde{N}_{R}^{*}+2 \tilde{E}_{L} \tilde{E}_{L}^{*}-2 \tilde{\tau}_{R} \tilde{\tau}_{R}^{*}-2 \tilde{\mu}_{R} \tilde{\mu}_{R}^{*}-2 \tilde{e}_{R} \tilde{e}_{R}^{*}\right\}
\end{aligned}
$$


For $\mathcal{L}_{\text {soft }}$ we assume the following form

$$
\begin{aligned}
-\mathcal{L}_{\mathrm{soft}} & =\tilde{M}_{\tau L}^{2} \tilde{\psi}_{\tau L}^{i *} \tilde{\psi}_{\tau L}^{i}+\tilde{M}_{\chi}^{2} \tilde{\chi}^{c i *} \tilde{\chi}^{c i}+\tilde{M}_{\mu L}^{2} \tilde{\psi}_{\mu L}^{i *} \tilde{\psi}_{\mu L}^{i}+\tilde{M}_{e L}^{2} \tilde{\psi}_{e L}^{i *} \tilde{\psi}_{e L}^{i}+\tilde{M}_{\nu_{\tau}}^{2} \tilde{\nu}_{\tau L}^{c *} \tilde{\nu}_{\tau L}^{c}+\tilde{M}_{\nu_{\mu}}^{2} \tilde{\nu}_{\mu L}^{c *} \tilde{\nu}_{\mu L}^{c} \\
& +\tilde{M}_{\nu_{e}}^{2} \tilde{\nu}_{e L}^{c *} \tilde{\nu}_{e L}^{c}+\tilde{M}_{\tau}^{2} \tilde{\tau}_{L}^{c *} \tilde{\tau}_{L}^{c}+\tilde{M}_{\mu}^{2} \tilde{\mu}_{L}^{c *} \tilde{\mu}_{L}^{c}+\tilde{M}_{e}^{2} \tilde{e}_{L}^{c *} \tilde{e}_{L}^{c}+\tilde{M}_{E}^{2} \tilde{E}_{L}^{*} \tilde{E}_{L}+\tilde{M}_{N}^{2} \tilde{N}_{L}^{*} \tilde{N}_{L} \\
& +\epsilon_{i j}\left\{f_{1} A_{\tau} H_{1}^{i} \tilde{\psi}_{\tau L}^{j} \tilde{\tau}_{L}^{c}-f_{1}^{\prime} A_{\nu_{\tau}} H_{2}^{i} \tilde{\psi}_{\tau L}^{j} \tilde{\nu}_{\tau L}^{c}+h_{1} A_{\mu} H_{1}^{i} \tilde{\psi}_{\mu L}^{j} \tilde{\mu}_{L}^{c}-h_{1}^{\prime} A_{\nu_{\mu}} H_{2}^{i} \tilde{\psi}_{\mu L}^{j} \tilde{\nu}_{\mu L}^{c}\right. \\
& \left.+h_{2} A_{e} H_{1}^{i} \tilde{\psi}_{e L}^{j} \tilde{e}_{L}^{c}-h_{2}^{\prime} A_{\nu_{e}} H_{2}^{i} \tilde{\psi}_{e L}^{j} \tilde{\nu}_{e L}^{c}+f_{2} A_{N} H_{1}^{i} \tilde{\chi}^{c j} \tilde{N}_{L}-f_{2}^{\prime} A_{E} H_{2}^{i} \tilde{\chi}^{c j} \tilde{E}_{L}+\text { H.c. }\right\}
\end{aligned}
$$

\section{Interactions of leptons, scalar neutrinos and charginos}

In this section we discuss the interactions in the mass diagonal basis involving charged leptons, sneutrinos and charginos. Thus we have

$$
-\mathcal{L}_{\tau-\tilde{\nu}-\chi^{-}}=\sum_{i=1}^{2} \sum_{j=1}^{8} \bar{\tau}_{\alpha}\left(C_{\alpha i j}^{L} P_{L}+C_{\alpha i j}^{R} P_{R}\right) \tilde{\chi}^{c i} \tilde{\nu}_{j}+\text { H.c. },
$$

such that

$$
\begin{aligned}
C_{\alpha i j}^{L}= & g\left(-\kappa_{\tau} U_{i 2}^{*} D_{R 1 \alpha}^{\tau *} \tilde{D}_{1 j}^{\nu}-\kappa_{\mu} U_{i 2}^{*} D_{R 3 \alpha}^{\tau *} \tilde{D}_{5 j}^{\nu}-\kappa_{e} U_{i 2}^{*} D_{R 4 \alpha}^{\tau *} \tilde{D}_{7 j}^{\nu}+U_{i 1}^{*} D_{R 2 \alpha}^{\tau *} \tilde{D}_{4 j}^{\nu}-\kappa_{N} U_{i 2}^{*} D_{R 2 \alpha}^{\tau *} \tilde{D}_{2 j}^{\nu}\right) \\
C_{\alpha i j}^{R}= & g\left(-\kappa_{\nu_{\tau}} V_{i 2} D_{L 1 \alpha}^{\tau *} \tilde{D}_{3 j}^{\nu}-\kappa_{\nu_{\mu}} V_{i 2} D_{L 3 \alpha}^{\tau *} \tilde{D}_{6 j}^{\nu}-\kappa_{\nu_{e}} V_{i 2} D_{L 4 \alpha}^{\tau *} \tilde{D}_{8 j}^{\nu}+V_{i 1} D_{L 1 \alpha}^{\tau *} \tilde{D}_{1 j}^{\nu}+V_{i 1} D_{L 3 \alpha}^{\tau *} \tilde{D}_{5 j}^{\nu}\right. \\
& \left.+V_{i 1} D_{L 4 \alpha}^{\tau *} \tilde{D}_{7 j}^{\nu}-\kappa_{E} V_{i 2} D_{L 2 \alpha}^{\tau *} \tilde{D}_{4 j}^{\nu}\right),
\end{aligned}
$$

with

$$
\begin{gathered}
\left(\kappa_{N}, \kappa_{\tau}, \kappa_{\mu}, \kappa_{e}\right)=\frac{\left(m_{N}, m_{\tau}, m_{\mu}, m_{e}\right)}{\sqrt{2} m_{W} \cos \beta}, \\
\left(\kappa_{E}, \kappa_{\nu_{\tau}}, \kappa_{\nu_{\mu}}, \kappa_{\nu_{e}}\right)=\frac{\left(m_{E}, m_{\nu_{\tau}}, m_{\nu_{\mu}}, m_{\nu_{e}}\right)}{\sqrt{2} m_{W} \sin \beta} .
\end{gathered}
$$

\section{Interactions of leptons, sleptons and neutralinos}

In this section we discuss the interactions in the mass diagonal basis involving charged leptons, sleptons and neutralinos. Thus we have

$$
-\mathcal{L}_{\tau-\tilde{\tau}-\chi^{0}}=\sum_{i=1}^{4} \sum_{j=1}^{8} \bar{\tau}_{\alpha}\left(C_{\alpha i j}^{\prime L} P_{L}+C_{\alpha i j}^{\prime R} P_{R}\right) \tilde{\chi}_{i}^{0} \tilde{\tau}_{j}+\text { H.c. }
$$


such that

$$
\begin{aligned}
C_{\alpha i j}^{\prime L}= & \sqrt{2}\left(\alpha_{\tau i} D_{R 1 \alpha}^{\tau *} \tilde{D}_{1 j}^{\tau}-\delta_{E i} D_{R 2 \alpha}^{\tau *} \tilde{D}_{2 j}^{\tau}-\gamma_{\tau i} D_{R 1 \alpha}^{\tau *} \tilde{D}_{3 j}^{\tau}+\beta_{E i} D_{R 2 \alpha}^{\tau *} \tilde{D}_{4 j}^{\tau}+\alpha_{\mu i} D_{R 3 \alpha}^{\tau *} \tilde{D}_{5 j}^{\tau}-\gamma_{\mu i} D_{R 3 \alpha}^{\tau *} \tilde{D}_{6 j}^{\tau}\right. \\
& \left.+\alpha_{e i} D_{R 4 \alpha}^{\tau *} \tilde{D}_{7 j}^{\tau}-\gamma_{e i} D_{R 4 \alpha}^{\tau *} \tilde{D}_{8 j}^{\tau}\right) \\
C_{\alpha i j}^{\prime R}= & \sqrt{2}\left(\beta_{\tau i} D_{L 1 \alpha}^{\tau *} \tilde{D}_{1 j}^{\tau}-\gamma_{E i} D_{L 2 \alpha}^{\tau *} \tilde{D}_{2 j}^{\tau}-\delta_{\tau i} D_{L 1 \alpha}^{\tau *} \tilde{D}_{3 j}^{\tau}+\alpha_{E i} D_{L 2 \alpha}^{\tau *} \tilde{D}_{4 j}^{\tau}+\beta_{\mu i} D_{L 3 \alpha}^{\tau *} \tilde{D}_{5 j}^{\tau}-\delta_{\mu i} D_{L 3 \alpha}^{\tau *} \tilde{D}_{6 j}^{\tau}\right. \\
& \left.+\beta_{e i} D_{L 4 \alpha}^{\tau *} \tilde{D}_{7 j}^{\tau}-\delta_{e i} D_{L 4 \alpha}^{\tau *} \tilde{D}_{8 j}^{\tau}\right),
\end{aligned}
$$

where

$$
\begin{array}{ll}
\alpha_{E i}=\frac{g m_{E} X_{4 i}^{*}}{2 m_{W} \sin \beta} ; & \beta_{E i}=e X_{1 i}^{\prime}+\frac{g}{\cos \theta_{W}} X_{2 i}^{\prime}\left(\frac{1}{2}-\sin ^{2} \theta_{W}\right) \\
\gamma_{E i}=e X_{1 i}^{\prime *}-\frac{g \sin ^{2} \theta_{W}}{\cos \theta_{W}} X_{2 i}^{\prime *} ; & \delta_{E i}=-\frac{g m_{E} X_{4 i}}{2 m_{W} \sin \beta}
\end{array}
$$

and

$$
\begin{aligned}
& \alpha_{\tau i}=\frac{g m_{\tau} X_{3 i}}{2 m_{W} \cos \beta} ; \quad \alpha_{\mu i}=\frac{g m_{\mu} X_{3 i}}{2 m_{W} \cos \beta} ; \quad \alpha_{e i}=\frac{g m_{e} X_{3 i}}{2 m_{W} \cos \beta} \\
& \delta_{\tau i}=-\frac{g m_{\tau} X_{3 i}^{*}}{2 m_{W} \cos \beta} ; \quad \delta_{\mu i}=-\frac{g m_{\mu} X_{3 i}^{*}}{2 m_{W} \cos \beta} ; \quad \delta_{e i}=-\frac{g m_{e} X_{3 i}^{*}}{2 m_{W} \cos \beta}
\end{aligned}
$$

and where

$$
\begin{aligned}
& \beta_{\tau i}=\beta_{\mu i}=\beta_{e i}=-e X_{1 i}^{\prime *}+\frac{g}{\cos \theta_{W}} X_{2 i}^{*}\left(-\frac{1}{2}+\sin ^{2} \theta_{W}\right) \\
& \gamma_{\tau i}=\gamma_{\mu i}=\gamma_{e i}=-e X_{1 i}^{\prime}+\frac{g \sin ^{2} \theta_{W}}{\cos \theta_{W}} X_{2 i}^{\prime}
\end{aligned}
$$

Here $X^{\prime}$ are defined by

$$
\begin{aligned}
& X_{1 i}^{\prime}=X_{1 i} \cos \theta_{W}+X_{2 i} \sin \theta_{W} \\
& X_{2 i}^{\prime}=-X_{1 i} \sin \theta_{W}+X_{2 i} \cos \theta_{W}
\end{aligned}
$$

where $X$ diagonalizes the neutralino mass matrix, i.e.,

$$
X^{T} M_{\chi^{0}} X=\operatorname{diag}\left(m_{\chi_{1}^{0}}, m_{\chi_{2}^{0}}, m_{\chi_{3}^{0}}, m_{\chi_{4}^{0}}\right) .
$$




\section{Interaction of leptons and mirrors with $\mathrm{W}$ and $\mathrm{Z}$ bosons}

In addition to the computation of the supersymmetric loop diagrams, we compute the contributions arising from the exchange of the $\mathrm{W}$ and $\mathrm{Z}$ bosons and the leptons and the mirror leptons in the loops. The relevant interactions needed are given below. For the $\mathrm{W}$ boson exchange the interactions that enter are given by

$$
-\mathcal{L}_{\tau W \psi}=W_{\rho}^{\dagger} \sum_{i=1}^{4} \sum_{\alpha=1}^{4} \bar{\psi}_{i} \gamma^{\rho}\left[C_{L_{i \alpha}}^{W} P_{L}+C_{R_{i \alpha}}^{W} P_{R}\right] \tau_{\alpha}+\text { H.c. }
$$

where

$$
\begin{array}{r}
C_{L_{i \alpha}}^{W}=\frac{g}{\sqrt{2}}\left[D_{L 1 i}^{\nu *} D_{L 1 \alpha}^{\tau}+D_{L 3 i}^{\nu *} D_{L 3 \alpha}^{\tau}+D_{L 4 i}^{\nu *} D_{L 4 \alpha}^{\tau}\right] \\
C_{R_{i \alpha}}^{W}=\frac{g}{\sqrt{2}}\left[D_{R 2 i}^{\nu *} D_{R 2 \alpha}^{\tau}\right]
\end{array}
$$

For the $\mathrm{Z}$ boson exchange the interactions that enter are given by

$$
-\mathcal{L}_{\tau \tau Z}=Z_{\rho} \sum_{\alpha=1}^{4} \sum_{\beta=1}^{4} \bar{\tau}_{\alpha} \gamma^{\rho}\left[C_{L_{\alpha \beta}}^{Z} P_{L}+C_{R_{\alpha \beta}}^{Z} P_{R}\right] \tau_{\beta}
$$

where

$$
\begin{array}{r}
C_{L_{\alpha \beta}}^{Z}=\frac{g}{\cos \theta_{W}}\left[x\left(D_{L \alpha 1}^{\tau \dagger} D_{L 1 \beta}^{\tau}+D_{L \alpha 2}^{\tau \dagger} D_{L 2 \beta}^{\tau}+D_{L \alpha 3}^{\tau \dagger} D_{L 3 \beta}^{\tau}+D_{L \alpha 4}^{\tau \dagger} D_{L 4 \beta}^{\tau}\right)\right. \\
\left.-\frac{1}{2}\left(D_{L \alpha 1}^{\tau \dagger} D_{L 1 \beta}^{\tau}+D_{L \alpha 3}^{\tau \dagger} D_{L 3 \beta}^{\tau}+D_{L \alpha 4}^{\tau \dagger} D_{L 4 \beta}^{\tau}\right)\right]
\end{array}
$$

and

$$
\begin{array}{r}
C_{R_{\alpha \beta}}^{Z}=\frac{g}{\cos \theta_{W}}\left[x\left(D_{R \alpha 1}^{\tau \dagger} D_{R 1 \beta}^{\tau}+D_{R \alpha 2}^{\tau \dagger} D_{R 2 \beta}^{\tau}+D_{R \alpha 3}^{\tau \dagger} D_{R 3 \beta}^{\tau}+D_{R \alpha 4}^{\tau \dagger} D_{R 4 \beta}^{\tau}\right)\right. \\
\left.-\frac{1}{2}\left(D_{R \alpha 2}^{\tau \dagger} D_{R 2 \beta}^{\tau}\right)\right]
\end{array}
$$

where $x=\sin ^{2} \theta_{W}$.

\section{An analytical computation of the anomalous magnetic moment}

Using the interactions given in Section 3 the chargino contribution arises from the left diagram of Fig. 1. It is given by 

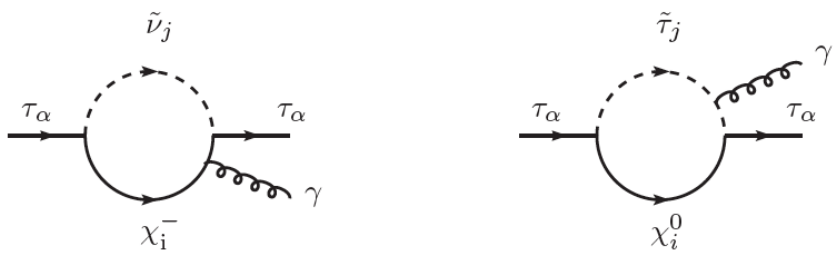

Figure 1: The diagrams that contribute to the leptonic $\left(\tau_{\alpha}\right)$ magnetic dipole moment via exchange of charginos $\left(\chi_{i}^{-}\right)$, sneutrinos and mirror sneutrinos $\left(\tilde{\nu}_{j}\right)$ (left diagram) inside the loop and from the exchange of neutralinos $\left(\chi_{i}^{0}\right)$ sleptons and mirror sleptons $\left(\tilde{\tau}_{j}\right)$ (right diagram) inside the loop.

$$
\begin{aligned}
a_{\alpha}^{\chi^{+}} & =-\sum_{i=1}^{2} \sum_{j=1}^{8} \frac{m_{\tau_{\alpha}}}{16 \pi^{2} m_{\chi_{i}^{+}}} \operatorname{Re}\left(C_{\alpha i j}^{L} C_{\alpha i j}^{R *}\right) F_{3}\left(\frac{m_{\tilde{\nu}_{j}}^{2}}{m_{\chi_{i}^{-}}^{2}}\right) \\
& +\sum_{i=1}^{2} \sum_{j=1}^{8} \frac{m_{\tau_{\alpha}}^{2}}{96 \pi^{2} m_{\chi_{i}^{+}}^{2}}\left[\left|C_{\alpha i j}^{L}\right|^{2}+\left|C_{\alpha i j}^{R}\right|^{2}\right] F_{4}\left(\frac{m_{\tilde{\nu}_{j}}^{2}}{m_{\chi_{i}^{-}}^{2}}\right),
\end{aligned}
$$

where the form factors $F_{3}$ and $F_{4}$ are given by

$$
F_{3}(x)=\frac{1}{(x-1)^{3}}\left[3 x^{2}-4 x+1-2 x^{2} \ln x\right]
$$

and

$$
F_{4}(x)=\frac{1}{(x-1)^{4}}\left[2 x^{3}+3 x^{2}-6 x+1-6 x^{2} \ln x\right]
$$

Using the interactions given in Section 4 the neutralino contribution arises from the right diagram of Fig. 1. It is given by

$$
\begin{aligned}
a_{\alpha}^{\chi^{0}} & =\sum_{i=1}^{4} \sum_{j=1}^{8} \frac{m_{\tau_{\alpha}}}{16 \pi^{2} m_{\chi_{i}^{0}}} \operatorname{Re}\left(C_{\alpha i j}^{\prime L} C_{\alpha i j}^{\prime R *}\right) F_{1}\left(\frac{m_{\tilde{\tau}_{j}}^{2}}{m_{\chi_{i}^{0}}^{2}}\right) \\
& +\sum_{i=1}^{2} \sum_{j=1}^{8} \frac{m_{\tau_{\alpha}}^{2}}{96 \pi^{2} m_{\chi_{i}^{0}}^{2}}\left[\left|C_{\alpha i j}^{\prime} L\right|^{2}+\left|C_{\alpha i j}^{\prime R}\right|^{2}\right] F_{2}\left(\frac{m_{\tilde{\tau}_{j}}^{2}}{m_{\chi_{i}^{0}}^{2}}\right)
\end{aligned}
$$

where the form factors are 


$$
F_{1}(x)=\frac{1}{(x-1)^{3}}\left[1-x^{2}+2 x \ln x\right]
$$

and

$$
F_{2}(x)=\frac{1}{(x-1)^{4}}\left[-x^{3}+6 x^{2}-3 x-2-6 x \ln x\right]
$$

The anomalous magnetic moments are known to exhibit a sharp dependence on the CP phases [22, 35. The dependence of $a_{e}$ on CP phases will be exhibited in the numerical analysis to follow.

The contributions to the lepton magnetic moment from the $\mathrm{W}$ and $\mathrm{Z}$ exchange arise from the diagrams of Fig. 2, Using the interactions given in Section 5 the contribution arising from the $\mathrm{W}$ exchange diagram (the left diagram of Fig. 2) is given by

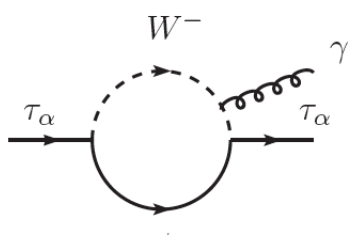

$\psi_{i}$

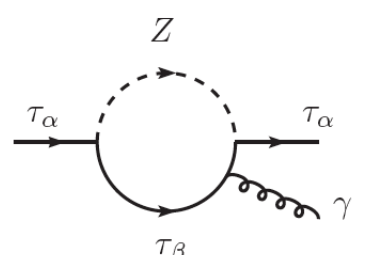

$\tau_{\beta}$

Figure 2: The W loop (the left diagram) involving the exchange of sequential and vectorlike neutrinos $\psi_{i}$ and the $\mathrm{Z}$ loop (the right diagram) involving the exchange of sequential and vectorlike charged leptons $\tau_{\beta}$ that contribute to the magnetic moment of the charged lepton $\tau_{\alpha}$.

$$
a_{\tau_{\alpha}}^{W}=\frac{m_{\tau_{\alpha}}^{2}}{16 \pi^{2} m_{W}^{2}} \sum_{i=1}^{4}\left[\left|C_{L i \alpha}^{W}\right|^{2}+\left|C_{R i \alpha}^{W}\right|^{2}\right] F_{W}\left(\frac{m_{\psi_{i}}^{2}}{m_{W}^{2}}\right)+\frac{m_{\psi_{i}}}{m_{\tau_{\alpha}}} \operatorname{Re}\left(C_{L i \alpha}^{W} C_{R i \alpha}^{W *}\right) G_{W}\left(\frac{m_{\psi_{i}}^{2}}{m_{W}^{2}}\right),
$$

where the form factors are given by

$$
F_{W}(x)=\frac{1}{6(x-1)^{4}}\left[4 x^{4}-49 x^{3}+18 x^{3} \ln x+78 x^{2}-43 x+10\right]
$$

and

$$
G_{W}(x)=\frac{1}{(x-1)^{3}}\left[4-15 x+12 x^{2}-x^{3}-6 x^{2} \ln x\right]
$$

Using the interactions given in Section 5 the contribution arising from the $\mathrm{Z}$ exchange diagram (the right diagram of Fig. 2) is given by 


$$
a_{\tau_{\alpha}}^{Z}=\frac{m_{\tau_{\alpha}}^{2}}{32 \pi^{2} m_{Z}^{2}} \sum_{\beta=1}^{4}\left[\left|C_{L \beta \alpha}^{Z}\right|^{2}+\left|C_{R \beta \alpha}^{Z}\right|^{2}\right] F_{Z}\left(\frac{m_{\tau_{\beta}}^{2}}{m_{Z}^{2}}\right)+\frac{m_{\tau_{\beta}}}{m_{\tau_{\alpha}}} \operatorname{Re}\left(C_{L \beta \alpha}^{Z} C_{R \beta \alpha}^{Z *}\right) G_{Z}\left(\frac{m_{\tau_{\beta}}^{2}}{m_{Z}^{2}}\right),
$$

where

$$
F_{Z}(x)=\frac{1}{3(x-1)^{4}}\left[-5 x^{4}+14 x^{3}-39 x^{2}+18 x^{2} \ln x+38 x-8\right]
$$

and

$$
G_{Z}(x)=\frac{2}{(x-1)^{3}}\left[x^{3}+3 x-6 x \ln x-4\right] .
$$

We now show that the standard model result [36] can be gotten in the limit when the off diagonal elements in the neutrino and lepton mass matrices are set to zero. The $\mathrm{W}$ boson contribution is obtained in this case where the couplings are $C_{L i \alpha}^{W}=\frac{g}{\sqrt{2}}$ for $i=\alpha$ and zero othewise and $C_{R i \alpha}^{W}=0$. In this limit, the form factor $F_{W}(0)=\frac{5}{3}$ and one gets

$$
a_{\tau_{\alpha}}^{W}=\frac{5 g^{2} m_{\tau_{\alpha}}^{2}}{96 \pi^{2} m_{W}^{2}}
$$

Using the relation that $G_{F}=\frac{\pi \alpha_{e m}}{\sqrt{2} m_{W}^{2} \sin ^{2} \theta_{W}}$, one gets the well known $\mathrm{W}$ boson contribution to the lepton $\tau_{\alpha}$

$$
a_{\tau_{\alpha}}^{W}=\frac{5 G_{F} m_{\tau_{\alpha}}^{2}}{12 \sqrt{2} \pi^{2}}
$$

where $\alpha=3$ for the case of muon and $\alpha=4$ for the case of electron.

To recover the $\mathrm{Z}$ boson contribution in the standard model limit we set

$$
\begin{array}{r}
C_{L \beta \alpha}^{Z}=\frac{g}{\cos \theta_{W}}\left(x-\frac{1}{2}\right), \\
C_{R \beta \alpha}^{Z}=\frac{g}{\cos \theta_{W}} x
\end{array}
$$

for the case of $\alpha=\beta$ and are set to zero otherwise. The form factors in this limit are given by $F_{Z}(0)=-\frac{8}{3}$ and $G_{Z}(0)=8$. In this case one finds for the $\mathrm{Z}$ contribution, the well known Standard Model result

$$
a_{\tau_{\alpha}}^{Z}=\frac{G_{F} m_{\tau_{\alpha}}^{2}}{2 \sqrt{2} \pi^{2}}\left[-\frac{5}{12}+\frac{4}{3}\left(\sin ^{2} \theta_{W}-\frac{1}{4}\right)^{2}\right]
$$




\begin{tabular}{lcc}
\hline \hline & (i) Case of no mixing & (ii) Case of mixing \\
\cline { 2 - 3 } Chargino contribution & $1.52 \times 10^{-14}$ & $5.76 \times 10^{-13}$ \\
Neutralino contribution & $7.03 \times 10^{-16}$ & $4.47 \times 10^{-16}$ \\
W boson contribution & $9.09 \times 10^{-14}$ & $1.02 \times 10^{-13}$ \\
Z boson contribution & $-4.59 \times 10^{-14}$ & $-3.89 \times 10^{-14}$ \\
\hline$\Delta a_{e}(\mathrm{EW})$ total & $6.08 \times 10^{-14}$ & $6.39 \times 10^{-13}$ \\
\hline \hline
\end{tabular}

Table 1: An exhibition the relative contributions to the electron magnetic dipole moment arising from chargino exchange, neutralino exchange, $\mathrm{W}$ boson exchange and $\mathrm{Z}$ boson exchange and their sum for the case when (i) there is no mixing among generations and for the case when (ii) mixing occurs. The common parameters for the two cases are $m_{E}=250, m_{0}=m_{0}^{\tilde{\nu}}=650,\left|A_{0}\right|=520$, $\left|A_{0}^{\tilde{\nu}}\right|=650,|\mu|=102,\left|m_{1}\right|=600,\left|m_{2}\right|=680, \theta_{A_{0}}=1.2, \theta_{A_{0}^{\tilde{\nu}}}=2.8, \theta_{1}=2.5, \theta_{2}=1.5, \theta_{\mu}=0.5$, $m_{N}=212$ and $\tan \beta=15$. For case (i), the couplings $f_{3}=f_{3}^{\prime}=f_{3}^{\prime \prime}=f_{4}=f_{4}^{\prime}=f_{4}^{\prime \prime}=f_{5}=$ $f_{5}^{\prime}=f_{5}^{\prime \prime}=0$. For case (ii), the $\mathrm{f}$ couplings are non-zero and have the values $\left|f_{3}\right|=7 \times 10^{-8}$, $\left|f_{3}^{\prime}\right|=5 \times 10^{-8},\left|f_{3}^{\prime \prime}\right|=8 \times 10^{-9},\left|f_{4}\right|=\left|f_{4}^{\prime}\right|=10,\left|f_{4}^{\prime \prime}\right|=120,\left|f_{5}\right|=8.11 \times 10^{-2},\left|f_{5}^{\prime}\right|=9.8 \times 10^{-2}$, $\left|f_{5}^{\prime \prime}\right|=4 \times 10^{-2}$ and their phases are $\theta_{f_{3}}=0.3, \theta_{f_{3}^{\prime}}=0.2, \theta_{f_{3}^{\prime \prime}}=0.6, \theta_{f_{4}}=1.4, \theta_{f_{4}^{\prime}}=1.1, \theta_{f_{4}^{\prime \prime}}=0.5$, $\theta_{f_{5}}=1.9, \theta_{f_{5}^{\prime}}=0.5$ and $\theta_{f_{5}^{\prime \prime}}=0.7$. All masses are in GeV and phases in rad. A comparison of case (i) and case (ii) indicates a very significant increase for case (ii) overall. The last row gives the total electroweak contribution to the anomalous magnetic moment which is sum of the four contributions in rows 1-4. It is seen that the total contribution for case (ii) is 10.5 times larger than for case (i).

\section{Numerical analysis and results}

In this section we present a detailed numerical analysis of the effect of the extra vectorlike generation on the magnetic moment of the electron. We will also study the effects of CP phases on the electron magnetic moment. The analysis is done under the Brookhaven constraint on the anomalous magnetic moment of the muon, i.e., the constraint of Eq.(6). As evident from the discussion of Section 2, the analysis is carried out in an MSSM extension with soft breaking parameters taken at the electroweak scale. Thus no renormalization group running of GUT scale parameters is needed. The parameters entering the analysis are summarized in the Appendix. In Table 1, we give a comparative analysis for the values of the electron anomalous magnetic moment for the case where no mixing occurs between generations and the case where such mixing takes place.

For case (i) in Table 1 , the couplings $f_{3}, f_{3}^{\prime}, f_{3}^{\prime \prime}, f_{4}, f_{4}^{\prime}, f_{4}^{\prime \prime}, f_{5}, f_{5}^{\prime}$ and $f_{5}^{\prime \prime}$ are all set to zero and this represents the case of no mixing between the generations. The upper two rows exhibit the chargino and neutralino contributions to $a_{e}$ while the next two rows give the standard model contribution arising from $W$ and $Z$ exchanges given by Eqs. (58) and (60). The total $\Delta a_{e}(\mathrm{EW})$ is shown in the bottom row and in this case it is $\sim 6 \times 10^{-14}$. Case (ii) in Table 1 is when we include 
mixings between the vector generation and the sequential generations. Here the $f$-couplings listed above assume non-zero values as shown in the table caption, indicating mixing between generations. The rows exhibit the supersymmetric exchange contribution and the standard model contribution in same order as for case (i). The analysis shows that the total contribution from the electro-weak sector increases by a factor of over 10 in the case when the mixing of the standard model generations with the vector multiplet is taken into account. The total electroweak correction in this case is $\Delta a_{e}(\mathrm{EW})=6.39 \times 10^{-13}$ which lies just below the error corridor of Eq. (5). The sparticles that enter the loops are neutralinos and sleptons, and charginos and sneutralinos. The current experimental lower bound on the neutralino mass is $\sim 50 \mathrm{GeV}$ and on the chargino and on the slepton masses is $\sim 100 \mathrm{GeV}$ [21]. The analysis presented here respects these bounds.

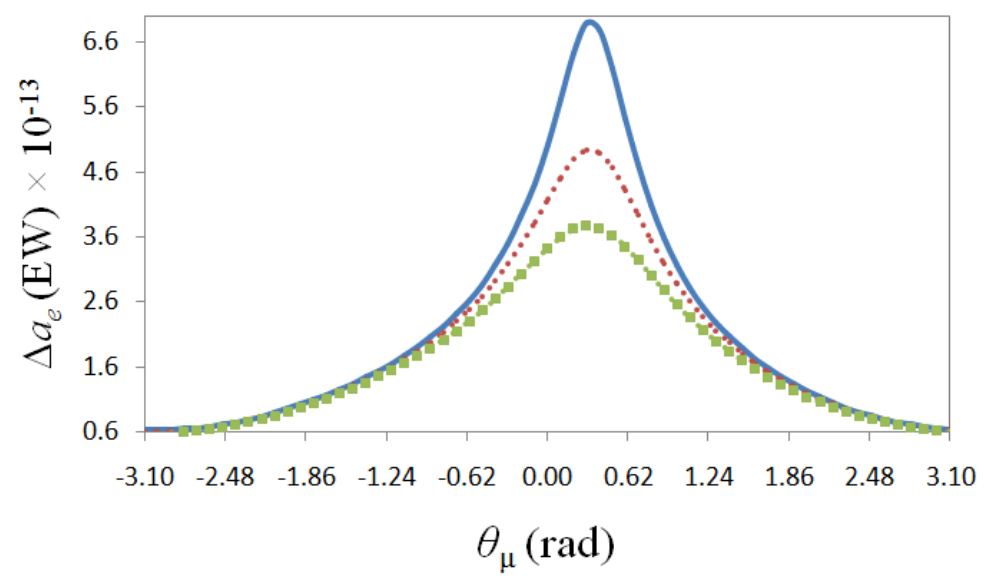

Figure 3: A display of the electron anomalous magnetic moment as a function of $\theta_{\mu}$, the phase of $\mu$, in the range $[-\pi, \pi]$. The three curves correspond to $m_{0}^{\tilde{\nu}}=650,\left|A_{0}^{\tilde{\nu}}\right|=650,\left|A_{0}\right|=520$ (solid curve), $m_{0}^{\tilde{\nu}}=660,\left|A_{0}^{\tilde{\nu}}\right|=655,\left|A_{0}\right|=530$ (dotted curve) and $m_{0}^{\tilde{\nu}}=675,\left|A_{0}^{\tilde{\nu}}\right|=660,\left|A_{0}\right|=540$ (square dotted curve). Other parameters have the values $m_{N}=212, m_{E}=250, m_{0}=650,\left|m_{1}\right|=600$, $\left|m_{2}\right|=240,|\mu|=104, \tan \beta=15,\left|f_{3}\right|=7 \times 10^{-8},\left|f_{3}^{\prime}\right|=5 \times 10^{-8},\left|f_{3}^{\prime \prime}\right|=8 \times 10^{-9},\left|f_{4}\right|=\left|f_{4}^{\prime}\right|=10$, $\left|f_{4}^{\prime \prime}\right|=90,\left|f_{5}\right|=8.11 \times 10^{-2},\left|f_{5}^{\prime}\right|=9.8 \times 10^{-2},\left|f_{5}^{\prime \prime}\right|=4 \times 10^{-2}, \theta_{A_{0}}=1.2, \theta_{A_{0}^{\tilde{\nu}}}=2.8, \theta_{1}=2.5$, $\theta_{2}=1.5, \theta_{f_{3}}=0.3, \theta_{f_{3}^{\prime}}=0.2, \theta_{f_{3}^{\prime \prime}}=0.6, \theta_{f_{4}}=1.4, \theta_{f_{4}^{\prime}}=1.1, \theta_{f_{4}^{\prime \prime}}=0.5, \theta_{f_{5}}=1.9, \theta_{f_{5}^{\prime}}=0.5$ and $\theta_{f_{5}^{\prime \prime}}=0.7$. All masses are in $\mathrm{GeV}$ and phases in rad.

It is known that the supersymmetric electroweak correction to the anomalous magnetic moment is sensitive to $\mathrm{CP}$ phases. This was demonstrated for the case of the supersymmetric electroweak contributions to the anomalous magnetic moment of the muon in [22]. Here we exhibit this sensitivity for the case of the electroweak contributions to the electron anomalous magnetic moment. 
Thus Figure 3 displays the total electroweak contribution to the anomalous magnetic moment of the electron as a function of $\theta_{\mu}$ which is the phase of $\mu$ that appears in the chargino and neutralino mass matrices and in the slepton and sneutrino mass ${ }^{2}$ matrices. Over the interval $[-\pi, \pi]$, the electroweak correction to the anomalous magnetic moment of the electron shows a pronounced peak for a value of $\theta_{\mu}=0.3 \mathrm{rad}$. For the three sets of values considered, the peak values stretch from $\sim 3.7-6.9 \times 10^{-13}$. It should be noted that the variation in $a_{e}$ comes from the supersymmetric sector, and mainly from the chargino contribution. This is so because the neutralino contribution is relatively small, typically an order of magnitude smaller than the chargino exchange contribution. Because of this the variation of $a_{e}$ with CP phases is dominated by the chargino contributions. We note also that the $W$ and $Z$ contributions are not affected by the phases.

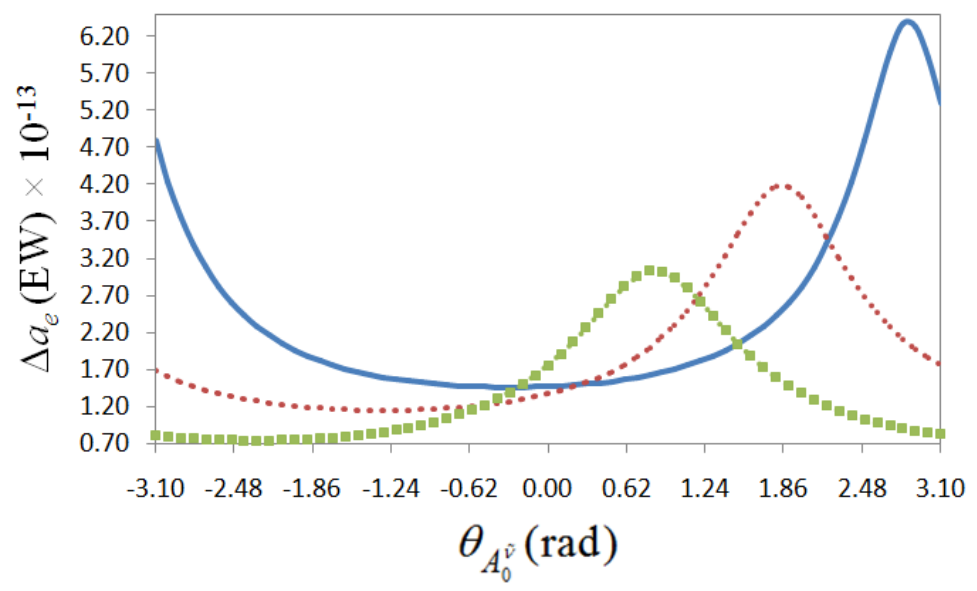

Figure 4: A display of the electroweak contribution to the anomalous magnetic moment of the electron as a function of $\theta_{A_{0}^{\tilde{\nu}}}$, the phase of $A_{0}^{\tilde{\nu}}$, in the range $[-\pi, \pi]$. The three curves correspond to (i) $m_{0}^{\tilde{\nu}}=650, \theta_{\mu}=0.3$ (solid curve), (ii) $m_{0}^{\tilde{\nu}}=660, \theta_{\mu}=1.3$ (dotted curve) and (iii) $m_{0}^{\tilde{\nu}}=670$, $\theta_{\mu}=2.3$ (square dotted curve). Other parameters have the values $m_{N}=212, m_{E}=250, m_{0}=650$, $\left|m_{1}\right|=600,\left|m_{2}\right|=240,|\mu|=103, \tan \beta=15,\left|A_{0}^{\tilde{\nu}}\right|=660,\left|A_{0}\right|=520,\left|f_{3}\right|=7 \times 10^{-8}$, $\left|f_{3}^{\prime}\right|=5 \times 10^{-8},\left|f_{3}^{\prime \prime}\right|=8 \times 10^{-9},\left|f_{4}\right|=\left|f_{4}^{\prime}\right|=10,\left|f_{4}^{\prime \prime}\right|=90,\left|f_{5}\right|=8.11 \times 10^{-2},\left|f_{5}^{\prime}\right|=9.8 \times 10^{-2}$, $\left|f_{5}^{\prime \prime}\right|=4 \times 10^{-2}, \theta_{A_{0}}=1.2, \theta_{1}=2.5, \theta_{2}=1.5, \theta_{f_{3}}=0.3, \theta_{f_{3}^{\prime}}=0.2, \theta_{f_{3}^{\prime \prime}}=0.6, \theta_{f_{4}}=1.4, \theta_{f_{4}^{\prime}}=1.1$, $\theta_{f_{4}^{\prime \prime}}=0.5, \theta_{f_{5}}=1.9, \theta_{f_{5}^{\prime}}=0.5$ and $\theta_{f_{5}^{\prime \prime}}=0.7$. All masses are in $\mathrm{GeV}$ and phases in rad.

Figure 4 exhibits the variation of electroweak contribution to the anomalous magnetic moment of the electron $\Delta a_{e}(\mathrm{EW})$ as a function of $\theta_{A_{0}^{\tilde{\nu}}}$, the phase of the trilinear coupling $A_{0}^{\tilde{\nu}}$, where in our analysis we have assumed that $A_{\nu_{\tau}}=A_{\nu_{\mu}}=A_{\nu_{e}}=A_{N}=A_{0}^{\tilde{\nu}}$ and $m_{0}^{\tilde{\nu}^{2}}=\tilde{M}_{N}^{2}=\tilde{M}_{\nu_{\tau}}^{2}=\tilde{M}_{\nu_{\mu}}^{2}=\tilde{M}_{\nu_{e}}^{2}$ in the sneutrino mass ${ }^{2}$ matrix (see Appendix). Note that $m_{0}^{2}=\tilde{M}_{\tau L}{ }^{2}=\tilde{M}_{E}^{2}=\tilde{M}_{\tau}^{2}=\tilde{M}_{\chi}^{2}=\tilde{M}_{\mu L}^{2}=$ 
$\tilde{M}_{\mu}^{2}=\tilde{M}_{e L}^{2}=\tilde{M}_{e}^{2}$ and $A_{0}=A_{\tau}=A_{E}=A_{\mu}=A_{e}$ in the slepton mass ${ }^{2}$ matrix (see Appendix). As can be seen from Fig. 4 the variation is very substantial with $\Delta a_{e}(\mathrm{EW})$ varying in the range $\sim 7 \times 10^{-14}-6 \times 10^{-13}$ which is an order of magnitude variation. As for the case of Fig. 3 the source of variation is the chargino exchange contribution once again. This is so because the chargino exchange diagram contains the sneutrino mass matrix in the loop which has a strong $A_{0}^{\tilde{\nu}}$ dependence.

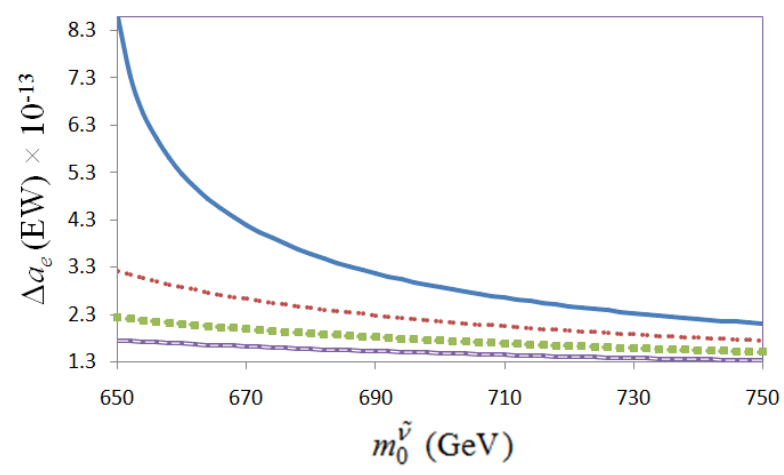

(a)

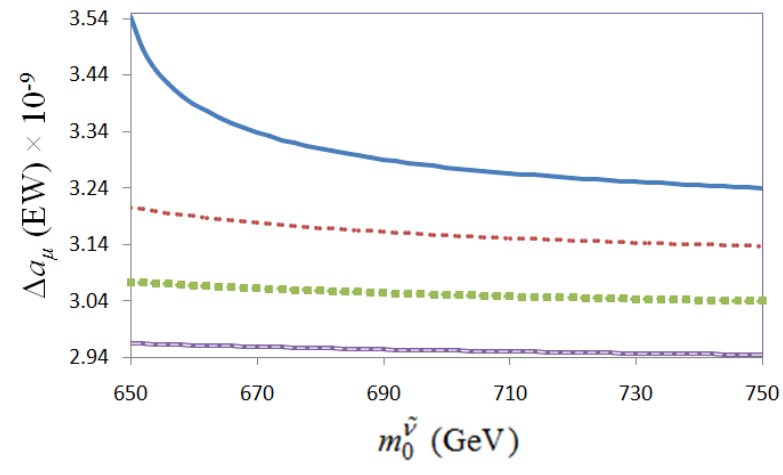

(b)

Figure 5: A display of the electroweak contribution to the anomalous magnetic moment of $e, \mu$ as a function of $m_{0}^{\tilde{\nu}}$ in the range $650-750 \mathrm{GeV}$. Panel (a) gives the electron anomalous magnetic moment and panel (b) gives the muon anomalous magnetic moment. The curves correspond to $\tan \beta=13$ (lowermost curve), $\tan \beta=14$ (square dotted curve), $\tan \beta=15$ (dotted curve), and $\tan \beta=16$ (solid curve). Other parameters have the values $m_{N}=212, m_{E}=250, m_{0}=650,\left|m_{1}\right|=600$, $\left|m_{2}\right|=200,|\mu|=104,\left|A_{0}^{\tilde{\nu}}\right|=650,\left|A_{0}\right|=520,\left|f_{3}\right|=7 \times 10^{-8},\left|f_{3}^{\prime}\right|=5 \times 10^{-8},\left|f_{3}^{\prime \prime}\right|=8 \times 10^{-9}$, $\left|f_{4}\right|=\left|f_{4}^{\prime}\right|=10,\left|f_{4}^{\prime \prime}\right|=90,\left|f_{5}\right|=8.11 \times 10^{-2},\left|f_{5}^{\prime}\right|=9.8 \times 10^{-2},\left|f_{5}^{\prime \prime}\right|=4 \times 10^{-2}, \theta_{A_{0}}=1.2$, $\theta_{A_{0}^{\tilde{\nu}}}=2.8, \theta_{1}=2.5, \theta_{2}=1.5, \theta_{\mu}=1.0, \theta_{f_{3}}=0.3, \theta_{f_{3}^{\prime}}=0.2, \theta_{f_{3}^{\prime \prime}}=0.6, \theta_{f_{4}}=1.4, \theta_{f_{4}^{\prime}}=1.1$, $\theta_{f_{4}^{\prime \prime}}=0.5, \theta_{f_{5}}=1.9, \theta_{f_{5}^{\prime}}=0.5$ and $\theta_{f_{5}^{\prime \prime}}=0.7$. All masses are in $\mathrm{GeV}$ and phases in rad.

Figure 5 exhibits the variation of the electroweak contribution to the anomalous magnetic moment of the electron and of the muon as a function of $m_{0}^{\tilde{\nu}}$ over the range $650-750 \mathrm{GeV}$. For parametric curves corresponding to $\tan \beta=13,14,15,16$ (from bottom to top) are shown for each of the panels. A comparison of panel (a) with panel (b) shows that $a_{e}$ exhibits a much larger sensitivity to $m_{0}^{\tilde{\nu}}$. 


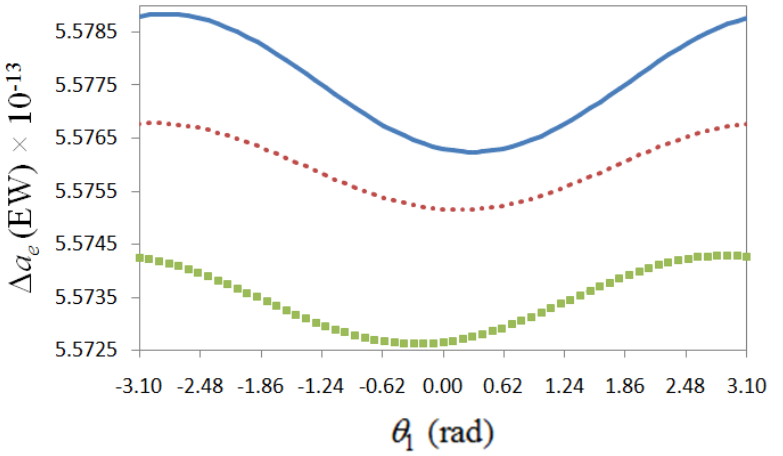

(a)

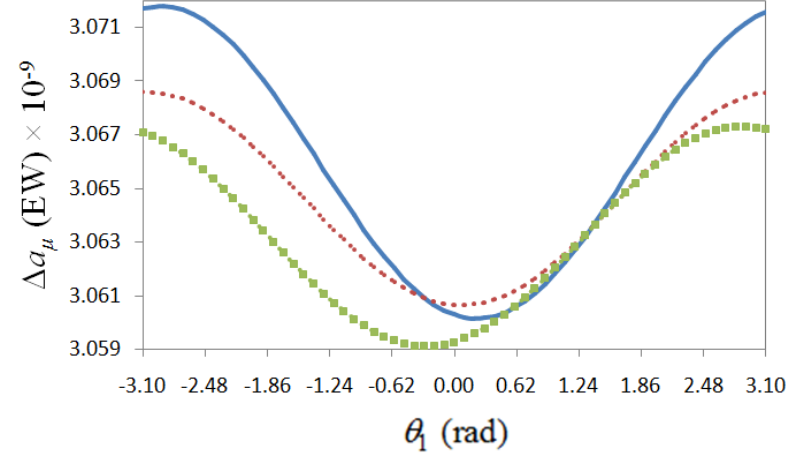

(b)

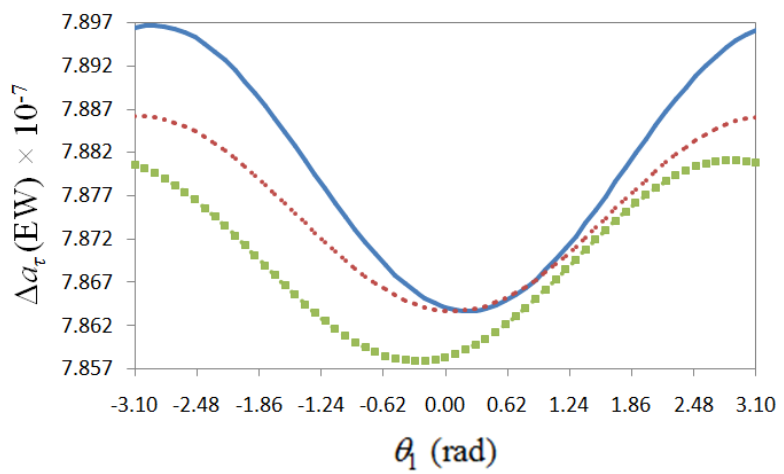

(c)

Figure 6: A display of the electroweak contribution to the anomalous magnetic moment of the $e, \mu, \tau$ as a function of $\theta_{1}$, the phase of $m_{1}$, in the range $[-\pi, \pi]$. Panel (a) gives the electroweak contribution to the anomalous magnetic moment of the electron, panel (b) gives it for the muon and panel (c) gives it for the tau. The curves correspond to (i) $\left|A_{0}\right|=520, \theta_{A_{0}}=1.2,\left|m_{2}\right|=350$ (solid curve), (ii) $\left|A_{0}\right|=330, \theta_{A_{0}}=1.9,\left|m_{2}\right|=351$ (dotted curve), and (iii) $\left|A_{0}\right|=130, \theta_{A_{0}}=2.6,\left|m_{2}\right|=352$ (lowermost curve). Other parameters have the values $m_{N}=212, m_{E}=250, m_{0}=m_{0}^{\tilde{\nu}}=650$, $\left|m_{1}\right|=600,|\mu|=104,\left|A_{0}^{\tilde{\nu}}\right|=640, \tan \beta=15,\left|f_{3}\right|=7 \times 10^{-8},\left|f_{3}^{\prime}\right|=5 \times 10^{-8},\left|f_{3}^{\prime \prime}\right|=8 \times 10^{-9}$, $\left|f_{4}\right|=\left|f_{4}^{\prime}\right|=10,\left|f_{4}^{\prime \prime}\right|=90,\left|f_{5}\right|=8.11 \times 10^{-2},\left|f_{5}^{\prime}\right|=9.8 \times 10^{-2},\left|f_{5}^{\prime \prime}\right|=4 \times 10^{-2}, \theta_{2}=0.1, \theta_{A_{0}^{\tilde{\nu}}}=2.8$, $\theta_{\mu}=0.3, \theta_{f_{3}}=0.3, \theta_{f_{3}^{\prime}}=0.2, \theta_{f_{3}^{\prime \prime}}=0.6, \theta_{f_{4}}=1.4, \theta_{f_{4}^{\prime}}=1.1, \theta_{f_{4}^{\prime \prime}}=0.5, \theta_{f_{5}}=1.9, \theta_{f_{5}^{\prime}}=0.5$ and $\theta_{f_{5}^{\prime \prime}}=0.7$. All masses are in $\mathrm{GeV}$ and phases in rad.

Figure 6 exhibits the variation of the electroweak contribution to the anomalous magnetic moment of the electron (panel (a)), of the muon (panel(b)) and of the tau (panel (c)) vs $\theta_{1}$, which is the phase of $m_{1}$, over the range $[-\pi, \pi]$. It seen that the variation is smooth in all cases as expected. However, the size of variation in each case is small as can be seen, for example, by comparing the range of variation in panel (a) in Fig. 6 with the range of variation in Fig. 3 . The 
reason for this smallness is easily understood. Thus the parameter $m_{1}$ enters in the neutralino mass matrix and as discussed earlier the contribution from the neutralino exchange diagram to the electroweak contribution to the anomalous magnetic moment of the electron is relatively small which explains the relative smallness of the variation of $\Delta a_{e}(\mathrm{EW})$ with $\theta_{1}$. Similar results hold for the variation of $\Delta a_{\mu}(\mathrm{EW})$ and $\Delta a_{\tau}(\mathrm{EW})$ with $\theta_{1}$. Regarding $a_{\tau}$ we note that the standard model predicts [37]

$$
a_{\tau}^{\mathrm{SM}}=117721(5) \times 10^{-8}
$$

The current experimental result is 38

$$
a_{\tau}^{\mathrm{EXP}}=-0.018(17)
$$

while the analysis of [39] constraints the range of new physics so that

$$
-0.007<\Delta a_{\tau}^{\mathrm{NP}}<0.005
$$

where $\Delta a_{\tau}^{\mathrm{NP}}$ refers to the new physics contribution. Future experiments [40] in high luminosity B factories are likely to significantly improve the limits in Eq. 63). However, it is unlikely that the improvements in the measurement of the tau anomalous magnetic moment at the level needed to check on the contributions of panel ( c) in Fig. 6 can be achieved in experiment in the very near future. Thus, $a_{e}$ gives the best hope for the test of new physics.

Next we investigate the limits on the parameter space arising from the constraints of Eq. (5) and Eq.(6). Using these we impose the following upper limit constraints on new physics contributions

$$
\begin{aligned}
& \Delta a_{e}^{\mathrm{NP}} \leq 8.2 \times 10^{-13} \\
& \Delta a_{\mu}^{\mathrm{NP}} \leq 2.87 \times 10^{-9}
\end{aligned}
$$

where $\Delta a_{e}^{\mathrm{NP}}$ stands for the new physics contribution to $a_{e}$ and $\Delta a_{\mu}^{\mathrm{NP}}$ stands for the new physics contribution to $a_{\mu}$. In Fig.(7) we give an analysis of the allowed (shaded) and excluded (empty) regions under the constraints of Eqs. 64, 65). Thus the left panel of Fig. (7) gives an analysis of the allowed parameter space in the $m_{0}-m_{2}$ plane of the constraint on the new physics contribution to the anomalous magnetic moment of the electron given by Eqs. (64) while the right panel of Fig.(7) gives an analysis of the allowed parameter space in the $m_{0}-m_{2}$ plane of the constraint on the new physics contribution to the anomalous magnetic moment of the muon given by Eqs. 65. We see that a part of the parameter space is excluded because of the constraints. The excluded regions are of course, sensitive to the other input parameters and different choices of those parameters 
would lead to modification of the allowed and the excluded regions. Next we exhibit the allowed and the excluded regions in the plane of CP phases. The analysis here is similar to the one in [41] done for the muon anomaly. Thus as noted earlier the anomalous magnetic moments are sensitive to $\mathrm{CP}$ phases and the constraint of Eq.(64) would have impact on the allowed regions of the CP phases. This is exhibited in Fig.(8) where the allowed (filled) and excluded (empty) regions under the constraint of Eq. 64 are exhibited in the plane of two phases: the phase of the Higgs mixing parameter $\theta_{\mu}$ and the phase of the trilinear coupling $A_{0}^{\tilde{\nu}}$, i.e., $\theta_{A_{0}^{\tilde{\nu}}}$. The analysis of Fig. (7) and Fig. (8) indicates that the constraints on new physics given by Eq.(64) and Eq.(65) have significant impact on the available parameter space of the extended MSSM model.

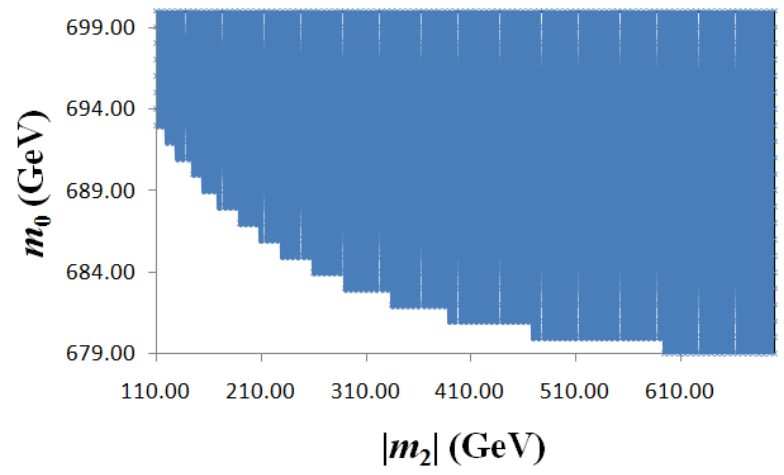

(a)

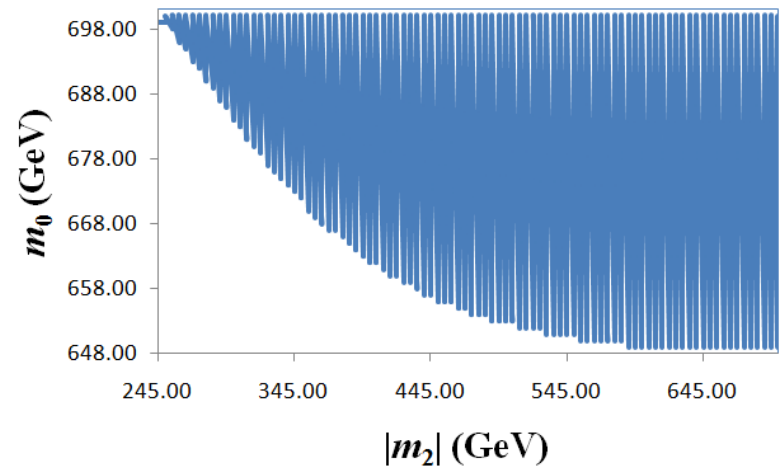

(b)

Figure 7: An exhibition of the allowed (shaded) and excluded (empty) regions in the $m_{0}-m_{2}$ plane under the anomalous magnetic moment constraints. Panel (a) gives a display of the allowed and forbidden values of $m_{0}$ and $\left|m_{2}\right|$ under the constraint on $a_{e}$ given by Eq. 64) and panel (b) gives a display of the allowed and forbidden regions of $m_{0}$ and $\left|m_{2}\right|$ under the constraint on $a_{\mu}$ of Eq. 65). Other parameters have the values $\tan \beta=15, m_{N}=212, m_{E}=180,\left|m_{1}\right|=600$, $|\mu|=104,\left|A_{0}^{\tilde{\nu}}\right|=651, m_{0}^{\tilde{\nu}}=650,\left|A_{0}\right|=520,\left|f_{3}\right|=7 \times 10^{-8},\left|f_{3}^{\prime}\right|=5 \times 10^{-8},\left|f_{3}^{\prime \prime}\right|=8 \times 10^{-9}$, $\left|f_{4}\right|=\left|f_{4}^{\prime}\right|=10,\left|f_{4}^{\prime \prime}\right|=100,\left|f_{5}\right|=8.11 \times 10^{-2},\left|f_{5}^{\prime}\right|=9.8 \times 10^{-2},\left|f_{5}^{\prime \prime}\right|=4 \times 10^{-2}, \theta_{A_{0}}=1.2$, $\theta_{A_{0}^{\tilde{\nu}}}=2.8, \theta_{1}=2.5, \theta_{2}=0, \theta_{\mu}=0.3, \theta_{f_{3}}=0.3, \theta_{f_{3}^{\prime}}=0.2, \theta_{f_{3}^{\prime \prime}}=0.6, \theta_{f_{4}}=1.4, \theta_{f_{4}^{\prime}}=1.1, \theta_{f_{4}^{\prime \prime}}=0.5$, $\theta_{f_{5}}=1.9, \theta_{f_{5}^{\prime}}=0.5$ and $\theta_{f_{5}^{\prime \prime}}=0.7$. All masses are in $\mathrm{GeV}$ and phases in rad. 


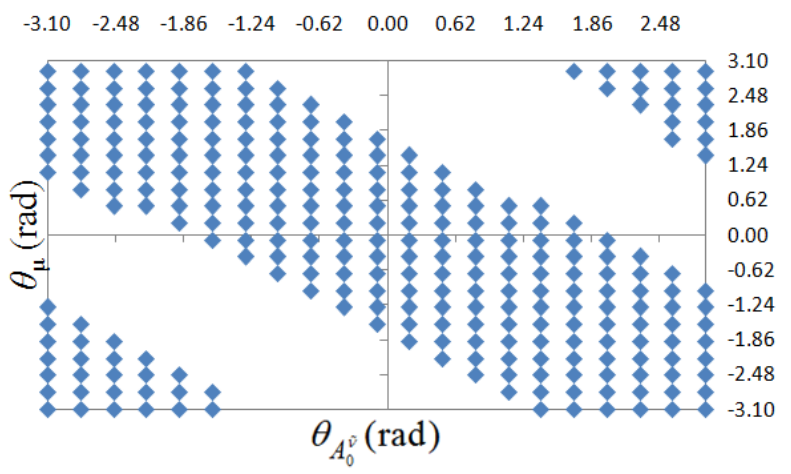

Figure 8: An exhibition of the allowed (shaded) and excluded (empty) regions in the $\theta_{\mu}-\theta_{A_{0}^{\tilde{\nu}}}$ plane under the constraint on $a_{e}$ given by Eq. (64). Other parameters have the values $\tan \beta=15$, $m_{N}=212, m_{E}=130, m_{0}=649,\left|m_{1}\right|=600,\left|m_{2}\right|=100,|\mu|=104,\left|A_{0}^{\tilde{\nu}}\right|=651, m_{0}^{\tilde{\nu}}=650$, $\left|A_{0}\right|=520,\left|f_{3}\right|=7 \times 10^{-8},\left|f_{3}^{\prime}\right|=5 \times 10^{-8},\left|f_{3}^{\prime \prime}\right|=8 \times 10^{-9},\left|f_{4}\right|=\left|f_{4}^{\prime}\right|=10,\left|f_{4}^{\prime \prime}\right|=140$, $\left|f_{5}\right|=8.11 \times 10^{-2},\left|f_{5}^{\prime}\right|=9.8 \times 10^{-2},\left|f_{5}^{\prime \prime}\right|=4 \times 10^{-2}, \theta_{A_{0}}=1.2, \theta_{1}=2.5, \theta_{2}=0, \theta_{f_{3}}=0.3$, $\theta_{f_{3}^{\prime}}=0.2, \theta_{f_{3}^{\prime \prime}}=0.6, \theta_{f_{4}}=1.4, \theta_{f_{4}^{\prime}}=1.1, \theta_{f_{4}^{\prime \prime}}=0.5, \theta_{f_{5}}=1.9, \theta_{f_{5}^{\prime}}=0.5$ and $\theta_{f_{5}^{\prime \prime}}=0.7$. All masses are in $\mathrm{GeV}$ and phases in rad.

\section{Conclusion}

The magnetic moment of the electron is one of the most precisely determined quantities in physics with an error in $a_{e}^{e x p}$ of $\delta a_{e}^{e x p}=2.8 \times 10^{-13}$. The theory predictions for $a_{e}$ have also been done with a high accuracy. However the error in theory prediction is significantly larger than the experimental error giving a total error in the difference in experiment minus theory of $\delta \Delta a_{e} \simeq 8 \times 10^{-13}$. This error is much larger than the new physics effects predicted by scaling if one extrapolates the discrepancy between experiment and theory for the muon anomalous magnetic moment. Thus the Brookhaven experiment gives $\left(a_{\mu}^{e x p}-a_{\mu}^{\text {theory }}\right)=(287 \pm 80) \times 10^{-11}$ and if one uses the scaling factor of $m_{e}^{2} / m_{\mu}^{2}$ the new physics effect in $a_{e}$ would be of size $(.6 \pm .2) \times 10^{-13}$ which is an order of magnitude smaller than the current error in $\delta \Delta a_{e}$. However, much larger new physics effects can occur if naive scaling law is violated. In this work we have shown that such violations do occur in extensions of MSSM with a vectorlike multiplet. In this regard we are in agreement with the conclusion of [15]). Thus we have computed the effect of both the non-supersymmetric as well as the supersymmetric loop corrections to the anomalous magnetic moment of the electron in the MSSM extension with a vectorlike multiplet. We have shown that effects as large as factors of five or more can occur in the MSSM extension over what one expects from scaling. We have also investigated the effect of $\mathrm{CP}$ phases on the correction from the new physics sector. The largeness of the correction opens the 
possibility that such effects could be discerned even with modest further improvement in reducing the error in $\Delta a_{e}$.

Acknowledgments: This research was supported in part by the NSF Grant PHY-1314774, XSEDETG-PHY110015, and NERSC-DE-AC02-05CH1123.

\section{Appendix: Further details on the scalar mass squared matrices}

In this Appendix we give further details of the structure of the slepton mass matrices. First we discuss briefly the parameters that enter the theory. The analysis we are doing is within the framework of an extended MSSM with soft parameters. Thus the analysis is done at the electroweak scale without renormalization group running. The soft sector of MSSM consists of the scalar slepton masses such as $m_{0}, U(1)(S U(2))$ gaugino masses $m_{1}\left(m_{2}\right)$ and trilinear couplings such as $A_{0}^{\tilde{\nu}}$ where $m_{1}\left(m_{2}\right), A_{0}^{\tilde{\nu}}$ are complex with phases $\theta_{1}, \theta_{2}, \theta_{A_{0}^{\tilde{\nu}}}$ etc. The other MSSM parameters include $\tan \beta=<H_{2}>/<H_{1}>$ where $H_{2}^{2}$ gives mass to the up quarks while $H_{1}^{1}$ gives mass to the down quarks and the leptons, and $\mu$ which is the Higgs mixing parameter which can also be complex with the phase $\theta_{\mu}$. The extended MSSM sector contains the vector lepton masses $m_{E}$, $m_{N}$, the mixing parameters defined by Eq.99) and soft parameters in the extended sector defined by Eq. (21). We give now further details. The mass terms arising from the superpotential are given by

$$
\mathcal{L}_{F}^{\text {mass }}=\mathcal{L}_{C}^{\text {mass }}+\mathcal{L}_{N}^{\text {mass }}
$$


where $\mathcal{L}_{C}^{\text {mass }}$ gives the mass terms for the charged leptons while $\mathcal{L}_{N}^{\text {mass }}$ gives the mass terms for the neutrinos. For $\mathcal{L}_{C}^{\text {mass }}$ we have

$$
\begin{aligned}
& -\mathcal{L}_{C}^{\text {mass }}=\left(\frac{v_{2}^{2}\left|f_{2}^{\prime}\right|^{2}}{2}+\left|f_{3}\right|^{2}+\left|f_{3}^{\prime}\right|^{2}+\left|f_{3}^{\prime \prime}\right|^{2}\right) \tilde{E}_{R} \tilde{E}_{R}^{*}+\left(\frac{v_{2}^{2}\left|f_{2}^{\prime}\right|^{2}}{2}+\left|f_{4}\right|^{2}+\left|f_{4}^{\prime}\right|^{2}+\left|f_{4}^{\prime \prime}\right|^{2}\right) \tilde{E}_{L} \tilde{E}_{L}^{*} \\
& +\left(\frac{v_{1}^{2}\left|f_{1}\right|^{2}}{2}+\left|f_{4}\right|^{2}\right) \tilde{\tau}_{R} \tilde{\tau}_{R}^{*}+\left(\frac{v_{1}^{2}\left|f_{1}\right|^{2}}{2}+\left|f_{3}\right|^{2}\right) \tilde{\tau}_{L} \tilde{\tau}_{L}^{*}+\left(\frac{v_{1}^{2}\left|h_{1}\right|^{2}}{2}+\left|f_{4}^{\prime}\right|^{2}\right) \tilde{\mu}_{R} \tilde{\mu}_{R}^{*} \\
& +\left(\frac{v_{1}^{2}\left|h_{1}\right|^{2}}{2}+\left|f_{3}^{\prime}\right|^{2}\right) \tilde{\mu}_{L} \tilde{\mu}_{L}^{*}+\left(\frac{v_{1}^{2}\left|h_{2}\right|^{2}}{2}+\left|f_{4}^{\prime \prime}\right|^{2}\right) \tilde{e}_{R} \tilde{e}_{R}^{*}+\left(\frac{v_{1}^{2}\left|h_{2}\right|^{2}}{2}+\left|f_{3}^{\prime \prime}\right|^{2}\right) \tilde{e}_{L} \tilde{e}_{L}^{*} \\
& +\left\{-\frac{f_{1} \mu^{*} v_{2}}{\sqrt{2}} \tilde{\tau}_{L} \tilde{\tau}_{R}^{*}-\frac{h_{1} \mu^{*} v_{2}}{\sqrt{2}} \tilde{\mu}_{L} \tilde{\mu}_{R}^{*}-\frac{f_{2}^{\prime} \mu^{*} v_{1}}{\sqrt{2}} \tilde{E}_{L} \tilde{E}_{R}^{*}+\left(\frac{f_{2}^{\prime} v_{2} f_{3}^{*}}{\sqrt{2}}+\frac{f_{4} v_{1} f_{1}^{*}}{\sqrt{2}}\right) \tilde{E}_{L} \tilde{\tau}_{L}^{*}\right. \\
& +\left(\frac{f_{4} v_{2} f_{2}^{\prime *}}{\sqrt{2}}+\frac{f_{1} v_{1} f_{3}^{*}}{\sqrt{2}}\right) \tilde{E}_{R} \tilde{\tau}_{R}^{*}+\left(\frac{f_{3}^{\prime} v_{2} f_{2}^{\prime *}}{\sqrt{2}}+\frac{h_{1} v_{1} f_{4}^{\prime *}}{\sqrt{2}}\right) \tilde{E}_{L} \tilde{\mu}_{L}^{*}+\left(\frac{f_{2}^{\prime} v_{2} f_{4}^{\prime *}}{\sqrt{2}}+\frac{f_{3}^{\prime} v_{1} h_{1}^{*}}{\sqrt{2}}\right) \tilde{E}_{R} \tilde{\mu}_{R}^{*} \\
& +\left(\frac{f_{3}^{\prime \prime *} v_{2} f_{2}^{\prime}}{\sqrt{2}}+\frac{f_{4}^{\prime \prime} v_{1} h_{2}^{*}}{\sqrt{2}}\right) \tilde{E}_{L} \tilde{e}_{L}^{*}+\left(\frac{f_{4}^{\prime \prime} v_{2} f_{2}^{\prime *}}{\sqrt{2}}+\frac{f_{3}^{\prime \prime *} v_{1} h_{2}^{*}}{\sqrt{2}}\right) \tilde{E}_{R} \tilde{e}_{R}^{*}+f_{3}^{\prime} f_{3}^{*} \tilde{\mu}_{L} \tilde{\tau}_{L}^{*}+f_{4} f_{4}^{\prime *} \tilde{\mu}_{R} \tilde{\tau}_{R}^{*} \\
& \left.+f_{4} f_{4}^{\prime \prime *} \tilde{e}_{R} \tilde{\tau}_{R}^{*}+f_{3}^{\prime \prime} f_{3}^{*} \tilde{e}_{L} \tilde{\tau}_{L}^{*}+f_{3}^{\prime \prime} f_{3}^{\prime *} \tilde{e}_{L} \tilde{\mu}_{L}^{*}+f_{4}^{\prime} f_{4}^{\prime \prime *} \tilde{e}_{R} \tilde{\mu}_{R}^{*}-\frac{h_{2} \mu^{*} v_{2}}{\sqrt{2}} \tilde{e}_{L} \tilde{e}_{R}^{*}+H . c .\right\}
\end{aligned}
$$

For $\mathcal{L}_{N}^{\text {mass }}$ we have

$$
\begin{gathered}
-\mathcal{L}_{N}^{\operatorname{mass}}=\left(\frac{v_{1}^{2}\left|f_{2}\right|^{2}}{2}+\left|f_{3}\right|^{2}+\left|f_{3}^{\prime}\right|^{2}+\left|f_{3}^{\prime \prime}\right|^{2}\right) \tilde{N}_{R} \tilde{N}_{R}^{*} \\
+\left(\frac{v_{1}^{2}\left|f_{2}\right|^{2}}{2}+\left|f_{5}\right|^{2}+\left|f_{5}^{\prime}\right|^{2}+\left|f_{5}^{\prime \prime}\right|^{2}\right) \tilde{N}_{L} \tilde{N}_{L}^{*}+\left(\frac{v_{2}^{2}\left|f_{1}^{\prime}\right|^{2}}{2}+\left|f_{5}\right|^{2}\right) \tilde{\nu}_{\tau R} \tilde{\nu}_{\tau R}^{*} \\
+\left(\frac{v_{2}^{2}\left|f_{1}^{\prime}\right|^{2}}{2}+\left|f_{3}\right|^{2}\right) \tilde{\nu}_{\tau L} \tilde{\nu}_{\tau L}^{*}+\left(\frac{v_{2}^{2}\left|h_{1}^{\prime}\right|^{2}}{2}+\left|f_{3}^{\prime}\right|^{2}\right) \tilde{\nu}_{\mu L} \tilde{\nu}_{\mu L}^{*}+\left(\frac{v_{2}^{2}\left|h_{1}^{\prime}\right|^{2}}{2}+\left|f_{5}^{\prime}\right|^{2}\right) \tilde{\nu}_{\mu R} \tilde{\nu}_{\mu R}^{*} \\
+\left(\frac{v_{2}^{2}\left|h_{2}^{\prime}\right|^{2}}{2}+\left|f_{3}^{\prime \prime}\right|^{2}\right) \tilde{\nu}_{e L} \tilde{\nu}_{e L}^{*}+\left(\frac{v_{2}^{2}\left|h_{2}^{\prime}\right|^{2}}{2}+\left|f_{5}^{\prime \prime}\right|^{2}\right) \tilde{\nu}_{e R} \tilde{\nu}_{e R}^{*} \\
+\left\{\begin{array}{c}
-\frac{f_{2} \mu^{*} v_{2}}{\sqrt{2}} \tilde{N}_{L} \tilde{N}_{R}^{*}-\frac{f_{1}^{\prime} \mu^{*} v_{1}}{\sqrt{2}} \tilde{\nu}_{\tau L} \tilde{\nu}_{\tau R}^{*}-\frac{h_{1}^{\prime} \mu^{*} v_{1}}{\sqrt{2}} \tilde{\nu}_{\mu L} \tilde{\nu}_{\mu R}^{*}+\left(\frac{f_{5} v_{2} f_{1}^{\prime *}}{\sqrt{2}}-\frac{f_{2} v_{1} f_{3}^{*}}{\sqrt{2}}\right) \tilde{N}_{L} \tilde{\nu}_{\tau L}^{*} \\
\left.\sqrt{2}-\frac{f_{1}^{\prime} v_{2} f_{3}^{*}}{\sqrt{2}}\right) \tilde{N}_{R} \tilde{\nu}_{\tau R}^{*}+\left(\frac{h_{1}^{\prime} v_{2} f_{5}^{\prime *}}{\sqrt{2}}-\frac{f_{3}^{\prime} v_{1} f_{2}^{*}}{\sqrt{2}}\right) \tilde{N}_{L} \tilde{\nu}_{\mu L}^{*}+\left(\frac{f_{5}^{\prime \prime} v_{1} f_{2}^{*}}{\sqrt{2}}-\frac{f_{3}^{\prime \prime *} v_{2} h_{2}^{\prime}}{\sqrt{2}}\right) \tilde{N}_{R} \tilde{\nu}_{e R}^{*} \\
+\left(\frac{h_{2}^{\prime *} v_{2} f_{5}^{\prime \prime}}{\sqrt{2}}-\frac{f_{3}^{\prime \prime *} v_{1} f_{2}}{\sqrt{2}}\right) \tilde{N}_{L} \tilde{\nu}_{e L}^{*}+\left(\frac{f_{5}^{\prime} v_{1} f_{2}^{*}}{\sqrt{2}}-\frac{h_{1}^{\prime} v_{2} f_{3}^{\prime *}}{\sqrt{2}}\right) \tilde{N}_{R} \tilde{\nu}_{\mu R}^{*} \\
+f_{3}^{\prime} f_{3}^{*} \tilde{\nu}_{\mu L} \tilde{\nu}_{\tau_{L}^{*}}+f_{5} f_{5}^{\prime *} \tilde{\nu}_{\mu R} \tilde{\nu}_{\tau R}^{*}-\frac{h_{2}^{\prime} \mu^{*} v_{1}}{\sqrt{2}} \tilde{\nu}_{e L} \tilde{\nu}_{e R}^{*} \\
+f_{3}^{\prime \prime} f_{3}^{*} \tilde{\nu}_{e L} \tilde{\nu}_{\tau L}^{*}+f_{5} f_{5}^{\prime \prime *} \tilde{\nu}_{e R} \tilde{\nu}_{\tau R}^{*}+f_{3}^{\prime \prime} f_{3}^{\prime *} \tilde{\nu}_{e L} \tilde{\nu}_{\mu L}^{*}+f_{5}^{\prime} f_{5}^{\prime \prime *} \tilde{\nu}_{e R} \tilde{\nu}_{\mu R}^{*}+H . c .
\end{array} .\right.
\end{gathered}
$$


We define the scalar mass squared matrix $M_{\tilde{\tau}}^{2}$ in the basis $\left(\tilde{\tau}_{L}, \tilde{E}_{L}, \tilde{\tau}_{R}, \tilde{E}_{R}, \tilde{\mu}_{L}, \tilde{\mu}_{R}, \tilde{e}_{L}, \tilde{e}_{R}\right)$. We label the matrix elements of these as $\left(M_{\tilde{\tau}}^{2}\right)_{i j}=M_{i j}^{2}$ where the elements of the matrix are given by

$$
\begin{aligned}
& M_{11}^{2}=\tilde{M}_{\tau L}^{2}+\frac{v_{1}^{2}\left|f_{1}\right|^{2}}{2}+\left|f_{3}\right|^{2}-m_{Z}^{2} \cos 2 \beta\left(\frac{1}{2}-\sin ^{2} \theta_{W}\right), \\
& M_{22}^{2}=\tilde{M}_{E}^{2}+\frac{v_{2}^{2}\left|f_{2}^{\prime}\right|^{2}}{2}+\left|f_{4}\right|^{2}+\left|f_{4}^{\prime}\right|^{2}+\left|f_{4}^{\prime \prime}\right|^{2}+m_{Z}^{2} \cos 2 \beta \sin ^{2} \theta_{W}, \\
& M_{33}^{2}=\tilde{M}_{\tau}^{2}+\frac{v_{1}^{2}\left|f_{1}\right|^{2}}{2}+\left|f_{4}\right|^{2}-m_{Z}^{2} \cos 2 \beta \sin ^{2} \theta_{W}, \\
& M_{44}^{2}=\tilde{M}_{\chi}^{2}+\frac{v_{2}^{2}\left|f_{2}^{\prime}\right|^{2}}{2}+\left|f_{3}\right|^{2}+\left|f_{3}^{\prime}\right|^{2}+\left|f_{3}^{\prime \prime}\right|^{2}+m_{Z}^{2} \cos 2 \beta\left(\frac{1}{2}-\sin ^{2} \theta_{W}\right), \\
& M_{55}^{2}=\tilde{M}_{\mu L}^{2}+\frac{v_{1}^{2}\left|h_{1}\right|^{2}}{2}+\left|f_{3}^{\prime}\right|^{2}-m_{Z}^{2} \cos 2 \beta\left(\frac{1}{2}-\sin ^{2} \theta_{W}\right), \\
& M_{66}^{2}=\tilde{M}_{\mu}^{2}+\frac{v_{1}^{2}\left|h_{1}\right|^{2}}{2}+\left|f_{4}^{\prime}\right|^{2}-m_{Z}^{2} \cos 2 \beta \sin ^{2} \theta_{W}, \\
& M_{77}^{2}=\tilde{M}_{e L}^{2}+\frac{v_{1}^{2}\left|h_{2}\right|^{2}}{2}+\left|f_{3}^{\prime \prime}\right|^{2}-m_{Z}^{2} \cos 2 \beta\left(\frac{1}{2}-\sin ^{2} \theta_{W}\right), \\
& M_{88}^{2}=\tilde{M}_{e}^{2}+\frac{v_{1}^{2}\left|h_{2}\right|^{2}}{2}+\left|f_{4}^{\prime \prime}\right|^{2}-m_{Z}^{2} \cos 2 \beta \sin ^{2} \theta_{W}, \\
& M_{12}^{2}=M_{21}^{2 *}=\frac{v_{2} f_{2}^{\prime} f_{3}^{*}}{\sqrt{2}}+\frac{v_{1} f_{4} f_{1}^{*}}{\sqrt{2}} \\
& M_{13}^{2}=M_{31}^{2 *}=\frac{f_{1}^{*}}{\sqrt{2}}\left(v_{1} A_{\tau}^{*}-\mu v_{2}\right), \\
& M_{14}^{2}=M_{41}^{2 *}=0, M_{15}^{2}=M_{51}^{2 *}=f_{3}^{\prime} f_{3}^{*}, \\
& M_{16}^{2 *}=M_{61}^{2 *}=0, M_{17}^{2 *}=M_{71}^{2 *}=f_{3}^{\prime \prime} f_{3}^{*}, M_{18}^{2 *}=M_{81}^{2 *}=0, M_{23}^{2}=M_{32}^{2 *}=0 \text {, } \\
& M_{24}^{2}=M_{42}^{2 *}=\frac{f_{2}^{\prime *}}{\sqrt{2}}\left(v_{2} A_{E}^{*}-\mu v_{1}\right), M_{25}^{2}=M_{52}^{2 *}=\frac{v_{2} f_{3}^{\prime} f_{2}^{\prime *}}{\sqrt{2}}+\frac{v_{1} h_{1} f_{4}^{*}}{\sqrt{2}}, \\
& M_{26}^{2}=M_{62}^{2 *}=0, M_{27}^{2}=M_{72}^{2 *}=\frac{v_{2} f_{3}^{\prime \prime} f_{2}^{\prime *}}{\sqrt{2}}+\frac{v_{1} h_{1} f_{4}^{\prime *}}{\sqrt{2}}, M_{28}^{2}=M_{82}^{2 *}=0, \\
& M_{34}^{2}=M_{43}^{2 *}=\frac{v_{2} f_{4} f_{2}^{\prime *}}{\sqrt{2}}+\frac{v_{1} f_{1} f_{3}^{*}}{\sqrt{2}}, M_{35}^{2}=M_{53}^{2 *}=0, M_{36}^{2}=M_{63}^{2 *}=f_{4} f_{4}^{\prime *}, \\
& M_{37}^{2}=M_{73}^{2 *}=0, M_{38}^{2}=M_{83}^{2 *}=f_{4} f_{4}^{\prime \prime *}, M_{45}^{2}=M_{54}^{2 *}=0, M_{46}^{2}=M_{64}^{2 *}=\frac{v_{2} f_{2}^{\prime} f_{4}^{\prime *}}{\sqrt{2}}+\frac{v_{1} f_{3}^{\prime} h_{1}^{*}}{\sqrt{2}}, \\
& M_{47}^{2}=M_{74}^{2 *}=0, M_{48}^{2}=M_{84}^{2 *}=\frac{v_{2} f_{2}^{\prime} f_{4}^{\prime \prime *}}{\sqrt{2}}+\frac{v_{1} f_{3}^{\prime \prime} h_{2}^{*}}{\sqrt{2}}, \\
& M_{56}^{2}=M_{65}^{2 *}=\frac{h_{1}^{*}}{\sqrt{2}}\left(v_{1} A_{\mu}^{*}-\mu v_{2}\right), M_{57}^{2}=M_{75}^{2 *}=f_{3}^{\prime \prime} f_{3}^{\prime *}, M_{58}^{2}=M_{85}^{2 *}=0, M_{67}^{2}=M_{76}^{2 *}=0, \\
& M_{68}^{2}=M_{86}^{2 *}=f_{4}^{\prime} f_{4}^{\prime \prime *}, M_{78}^{2}=M_{87}^{2 *}=\frac{h_{2}^{*}}{\sqrt{2}}\left(v_{1} A_{e}^{*}-\mu v_{2}\right)
\end{aligned}
$$


Here the terms $M_{11}^{2}, M_{13}^{2}, M_{31}^{2}, M_{33}^{2}$ arise from soft breaking in the sector $\tilde{\tau}_{L}, \tilde{\tau}_{R}$, the terms $M_{55}^{2}, M_{56}^{2}, M_{65}^{2}, M_{66}^{2}$ arise from soft breaking in the sector $\tilde{\mu}_{L}, \tilde{\mu}_{R}$, the terms $M_{77}^{2}, M_{78}^{2}, M_{87}^{2}, M_{88}^{2}$ arise from soft breaking in the sector $\tilde{e}_{L}, \tilde{e}_{R}$ and the terms $M_{22}^{2}, M_{24}^{2}, M_{42}^{2}, M_{44}^{2}$ arise from soft breaking in the sector $\tilde{E}_{L}, \tilde{E}_{R}$. The other terms arise from mixing between the staus, smuons and the mirrors. We assume that all the masses are of the electroweak size so all the terms enter in the mass squared matrix. We diagonalize this hermitian mass squared matrix by the unitary transformation $\tilde{D}^{\tau \dagger} M_{\tilde{\tau}}^{2} \tilde{D}^{\tau}=\operatorname{diag}\left(M_{\tilde{\tau}_{1}}^{2}, M_{\tilde{\tau}_{2}}^{2}, M_{\tilde{\tau}_{3}}^{2}, M_{\tilde{\tau}_{4}}^{2}, M_{\tilde{\tau}_{5}}^{2}, M_{\tilde{\tau}_{6}}^{2}, M_{\tilde{\tau}_{7}}^{2}, M_{\tilde{\tau}_{8}}^{2}\right)$. For a further clarification of the notation see [33].

The mass ${ }^{2}$ matrix in the sneutrino sector has a similar structure. In the basis $\left(\tilde{\nu}_{\tau L}, \tilde{N}_{L}\right.$, $\left.\tilde{\nu}_{\tau R}, \tilde{N}_{R}, \tilde{\nu}_{\mu L}, \tilde{\nu}_{\mu R}, \tilde{\nu}_{e L}, \tilde{\nu}_{e R}\right)$, we can write the sneutrino mass ${ }^{2}$ matrix in the form $\left(M_{\tilde{\nu}}^{2}\right)_{i j}=m_{i j}^{2}$ where

$$
\begin{gathered}
m_{11}^{2}=\tilde{M}_{\tau L}^{2}+m_{\nu_{\tau}}^{2}+\left|f_{3}\right|^{2}+\frac{1}{2} m_{Z}^{2} \cos 2 \beta, \\
m_{22}^{2}=\tilde{M}_{N}^{2}+m_{N}^{2}+\left|f_{5}\right|^{2}+\left|f_{5}^{\prime}\right|^{2}+\left|f_{5}^{\prime \prime}\right|^{2}, \\
m_{33}^{2}=\tilde{M}_{\nu_{\tau}}^{2}+m_{\nu_{\tau}}^{2}+\left|f_{5}\right|^{2}, \\
m_{44}^{2}=\tilde{M}_{\chi}^{2}+m_{N}^{2}+\left|f_{3}\right|^{2}+\left|f_{3}^{\prime}\right|^{2}+\left|f_{3}^{\prime \prime}\right|^{2}-\frac{1}{2} m_{Z}^{2} \cos 2 \beta, \\
m_{55}^{2}=\tilde{M}_{\mu L}^{2}+m_{\nu_{\mu}}^{2}+\left|f_{3}^{\prime}\right|^{2}+\frac{1}{2} m_{Z}^{2} \cos 2 \beta, \\
m_{66}^{2}=\tilde{M}_{\nu_{\mu}}^{2}+m_{\nu_{\mu}}^{2}+\left|f_{5}^{\prime}\right|^{2}, \\
m_{77}^{2}=\tilde{M}_{e L}^{2}+m_{\nu_{e}}^{2}+\left|f_{3}^{\prime \prime}\right|^{2}+\frac{1}{2} m_{Z}^{2} \cos 2 \beta \\
m_{88}^{2}=\tilde{M}_{\nu_{e}}^{2}+m_{\nu_{e}}^{2}+\left|f_{5}^{\prime \prime}\right|^{2}, \\
m_{12}^{2}=m_{21}^{2 *}=\frac{v_{2} f_{5} f_{1}^{\prime *}}{\sqrt{2}}-\frac{v_{1} f_{2} f_{3}^{*}}{\sqrt{2}}, \\
m_{13}^{2}=m_{31}^{2 *}=\frac{f_{1}^{\prime *}}{\sqrt{2}}\left(v_{2} A_{\nu_{\tau}}^{*}-\mu v_{1}\right), m_{14}^{2}=m_{41}^{2 *}=0, \\
m_{15}^{2}=m_{51}^{2 *}=f_{3}^{\prime} f_{3}^{*}, m_{16}^{2}=m_{61}^{2 *}=0, \\
m_{17}^{2}=m_{71}^{2 *}=f_{3}^{\prime \prime} f_{3}^{*}, m_{18}^{2}=m_{81}^{2 *}=0, \\
m_{23}^{2}=m_{32}^{2 *}=0, m_{24}^{2}=m_{42}^{2 *}=\frac{f_{2}^{*}}{\sqrt{2}}\left(v_{1} A_{N}^{*}-\mu v_{2}\right), m_{25}^{2}=m_{52}^{2 *}=-\frac{v_{1} f_{2}^{*} f_{3}^{\prime}}{\sqrt{2}}+\frac{h_{1}^{\prime} v_{2} f_{5}^{\prime *}}{\sqrt{2}} \\
m_{26}^{2}=m_{62}^{2 *}=0, m_{27}^{2}=m_{72}^{2 *}=-\frac{v_{1} f_{2}^{*} f_{3}^{\prime \prime}}{\sqrt{2}}+\frac{h_{2}^{\prime} v_{2} f_{5}^{\prime \prime *}}{\sqrt{2}},
\end{gathered}
$$




$$
\begin{gathered}
m_{28}^{2}=m_{82}^{2 *}=0, m_{34}^{2}=m_{43}^{2 *}=\frac{v_{1} f_{2}^{*} f_{5}}{\sqrt{2}}-\frac{v_{2} f_{1}^{\prime} f_{3}^{*}}{\sqrt{2}}, \\
m_{35}^{2}=m_{53}^{2 *}=0, m_{36}^{2}=m_{63}^{2 *}=f_{5} f_{5}^{\prime *}, m_{37}^{2}=m_{73}^{2 *}=0, m_{38}^{2}=m_{83}^{2 *}=f_{5} f_{5}^{\prime \prime *}, m_{45}^{2}=m_{54}^{2 *}=0, \\
m_{46}^{2}=m_{64}^{2 *}=-\frac{h_{1}^{\prime *} v_{2} f_{3}^{\prime}}{\sqrt{2}}+\frac{v_{1} f_{2} f_{5}^{\prime *}}{\sqrt{2}}, m_{47}^{2}=m_{74}^{2 *}=0, \\
m_{48}^{2}=m_{84}^{2 *}=\frac{v_{1} f_{2} f_{5}^{\prime \prime *}}{\sqrt{2}}-\frac{v_{2} h_{2}^{*} f_{3}^{\prime \prime}}{\sqrt{2}}, m_{56}^{2}=m_{65}^{2 *}=\frac{h_{1}^{\prime *}}{\sqrt{2}}\left(v_{2} A_{\nu_{\mu}}^{*}-\mu v_{1}\right), \\
m_{57}^{2}=m_{75}^{2 *}=f_{3}^{\prime \prime} f_{3}^{\prime *}, m_{58}^{2}=m_{85}^{2 *}=0, m_{67}^{2}=m_{76}^{2 *}=0, \\
m_{68}^{2}=m_{86}^{2 *}=f_{5}^{\prime} f_{5}^{\prime \prime *}, m_{78}^{2}=m_{87}^{2 *}=\frac{h_{2}^{\prime *}}{\sqrt{2}}\left(v_{2} A_{\nu_{e}}^{*}-\mu v_{1}\right) .
\end{gathered}
$$

As in the charged slepton sector here also the terms $m_{11}^{2}, m_{13}^{2}, m_{31}^{2}, m_{33}^{2}$ arise from soft breaking in the sector $\tilde{\nu}_{\tau L}, \tilde{\nu}_{\tau R}$, the terms $m_{55}^{2}, m_{56}^{2}, m_{65}^{2}, m_{66}^{2}$ arise from soft breaking in the sector $\tilde{\nu}_{\mu L}, \tilde{\nu}_{\mu_{R}}$, the terms $m_{77}^{2}, m_{78}^{2}, m_{87}^{2}, m_{88}^{2}$ arise from soft breaking in the sector $\tilde{\nu}_{e L}, \tilde{\nu}_{e R}$ and the terms $m_{22}^{2}, m_{24}^{2}, m_{42}^{2}, m_{44}^{2}$ arise from soft breaking in the sector $\tilde{N}_{L}, \tilde{N}_{R}$. The other terms arise

from mixing between the physical sector and the mirror sector. Again as in the charged lepton sector we assume that all the masses are of the electroweak size so all the terms enter in the mass $^{2}$ matrix. This mass ${ }^{2}$ matrix can be diagonalized by the unitary transformation $\tilde{D}^{\nu \dagger} M_{\tilde{\nu}}^{2} \tilde{D}^{\nu}=$ $\operatorname{diag}\left(M_{\tilde{\nu}_{1}}^{2}, M_{\tilde{\nu}_{2}}^{2}, M_{\tilde{\nu}_{3}}^{2}, M_{\tilde{\nu}_{4}}^{2}, M_{\tilde{\nu}_{5}}^{2}, M_{\tilde{\nu}_{6}}^{2}, M_{\tilde{\nu}_{7}}^{2}, M_{\tilde{\nu}_{8}}^{2}\right)$.

\section{References}

[1] D. Hanneke, S. Fogwell and G. Gabrielse, Phys. Rev. Lett. 100, 120801 (2008) arXiv:0801.1134 [physics.atom-ph]].

[2] T. Kinoshita and W.J. Marciano, in Quantum Electrodynamics, edited by T. Kinoshita, World Scientific (1990), pp. 419-478.

[3] J.S. Schwinger, Phys. Rev. 73 (1948) 416.

[4] C.M. Sommerfield, Phys. Rev. 107 (1957) 328; Ann. Phys. 5 (1958) 26; A. Petermann, Helv. Phys. Acta 30 (1957) 407; Nucl. Phys. 5 (1958) 677; H.H. Elend, Phys. Lett. 20 (1966) 682 [Erratum-ibid. 21 (1966) 720]; M. Passera, J. Phys. G 31 (2005) R75; P.J. Mohr, B.N. Taylor, D.B. Newell [CODATA 2010], arXiv:1203.5425 [physics.atom-ph];

[5] S. Laporta and E. Remiddi, Phys. Lett. B 379 (1996) 283; S. Laporta, Nuovo Cim. A 106 (1993) 675; S. Laporta and E. Remiddi, Phys. Lett. B 301 (1993) 440; M. Passera, Phys. 
Rev. D 75 (2007) 013002; M.A. Samuel and G. Li, Phys. Rev. D 44 (1991) 3935; 46 (1992) 4782(E); 48 (1993) 1879(E); S. Laporta and E. Remiddi, Nucl. Phys. Proc. Suppl. 181-182 (2008) 10.

[6] T. Aoyama, M. Hayakawa, T. Kinoshita and M. Nio, Phys. Rev. Lett. 99 (2007) 110406; Phys. Rev. D 77 (2008) 053012;

[7] A. L. Kataev, Phys. Rev. D 86, 013010 (2012) arXiv:1205.6191 [hep-ph]].

[8] T. Kinoshita and M. Nio, Phys. Rev. D 73 (2006) 053007; T. Aoyama, M. Hayakawa, T. Kinoshita and M. Nio, Nucl. Phys. B 740 (2006) 138; Nucl. Phys. B 796 (2008) 184; Phys. Rev. D 78 (2008) 113006; 82 (2010) 113004; 83 (2011) 053003; Phys. Rev. D 83 (2011) 053002; Phys. Rev. D 84 (2011) 053003; Phys. Rev. D 85 (2012) 033007; arXiv:1201.2461 [hep-ph]; T. Aoyama, M. Hayakawa, T. Kinoshita, M. Nio and N. Watanabe, Phys. Rev. D 78 (2008) 053005; T. Aoyama, K. Asano, M. Hayakawa, T. Kinoshita, M. Nio and N. Watanabe, Phys. Rev. D 81 (2010) 053009.

[9] T. Aoyama, M. Hayakawa, T. Kinoshita and M. Nio, Phys. Rev. Lett. 109, 111807 (2012) arXiv:1205.5368 [hep-ph]];

[10] T. Aoyama, M. Hayakawa, T. Kinoshita and M. Nio, Phys. Rev. Lett. 109, 111808 (2012) arXiv:1205.5370[hep-ph]].

[11] A. Kurz, T. Liu, P. Marquard and M. Steinhauser, Nucl. Phys. B 879, 1 (2014) arXiv:1311.2471 [hep-ph]].

[12] A. Czarnecki, B. Krause and W.J. Marciano, Phys. Rev. Lett. 76 (1996) 3267; Phys. Rev. D 52 (1995) 2619.

[13] D. Nomura and T. Teubner, arXiv:1208.4194 [hep-ph]; B. Krause, Phys. Lett. B 390 (1997) 392; J. Prades, E. de Rafael and A. Vainshtein, in "Lepton dipole moments," B.L. Roberts and W.J. Marciano (eds.), Advanced series on directions in high energy physics, Vol. 20, World Scientific (2010), pp. 303-317.

[14] F. Jegerlehner and A. Nyffeler, Phys. Rept. 477 (2009) 1.

[15] G. F. Giudice, P. Paradisi and M. Passera, JHEP 1211, 113 (2012) arXiv:1208.6583 [hep-ph]]. 
[16] R. Bouchendira, P. Clade, S. Guellati-Khelifa, F. Nez and F. Biraben, Phys. Rev. Lett. 106, 080801 (2011) [arXiv:1012.3627 [physics.atom-ph]].

[17] P. J. Mohr, B. N. Taylor and D. B. Newell, Rev. Mod. Phys. 84, 1527 (2012) arXiv:1203.5425 [physics.atom-ph]].

[18] K. Hagiwara, R. Liao, A. D. Martin, D. Nomura and T. Teubner, J. Phys. G 38, 085003 (2011) arXiv:1105.3149 [hep-ph]].

[19] M. Davier, A. Hoecker, B. Malaescu and Z. Zhang, Eur. Phys. J. C 71, 1515 (2011) [Erratumibid. C 72, 1874 (2012)] [arXiv:1010.4180 [hep-ph]].

[20] K. Kannike, M. Raidal, D. M. Straub and A. Strumia, JHEP 1202, 106 (2012) [Erratum-ibid. 1210, 136 (2012)] [arXiv:1111.2551 [hep-ph]].

[21] J. Beringer et al. (Particle Data Group), Phys. Rev. D86, 010001 (2012).

[22] T. Ibrahim and P. Nath, Phys. Rev. D 62, 015004 (2000) hep-ph/9908443]; Phys. Rev. D 61, 095008 (2000) hep-ph/9907555]; T. Ibrahim, U. Chattopadhyay and P. Nath, Phys. Rev. D 64, 016010 (2001) hep-ph/0102324]; For early works on supersymmetric contributions to the anomalous magnetic moments see T. C. Yuan, R. L. Arnowitt, A. H. Chamseddine and P. Nath, Z. Phys. C 26, 407 (1984); D. A. Kosower, L. M. Krauss and N. Sakai, Phys. Lett. B 133, 305 (1983). For a model independent approach see F. S. Queiroz and W. Shepherd, arXiv:1403.2309 [hep-ph].

[23] H. Georgi, Nucl. Phys. B 156, 126 (1979); F. Wilczek and A. Zee, Phys. Rev. D 25, 553 (1982); J. Maalampi, J.T. Peltoniemi, and M. Roos, PLB 220, 441(1989); J. Maalampi and M. Roos, Phys. Rept. 186, 53 (1990); K. S. Babu, I. Gogoladze, P. Nath and R. M. Syed, Phys. Rev. D 72, 095011 (2005) hep-ph/0506312]; Phys. Rev. D 74, 075004 (2006), arXiv:hepph/0607244]; Phys. Rev. D 85, 075002 (2012) arXiv:1112.5387 [hep-ph]]; P. Nath and R. M. Syed, Phys. Rev. D 81, 037701 (2010).

[24] K. S. Babu, I. Gogoladze, M. U. Rehman and Q. Shafi, Phys. Rev. D 78, 055017 (2008).

[25] C. Liu, Phys. Rev. D 80, 035004 (2009) [arXiv:0907.3011 [hep-ph]].

[26] S. P. Martin, Phys. Rev. D 81, 035004 (2010) arXiv:0910.2732 [hep-ph]]. 
[27] A. Aboubrahim, T. Ibrahim, A. Itani and P. Nath, Phys. Rev. D 89, 055009 (2014) arXiv:1312.2505 [hep-ph]].

[28] A. Aboubrahim, T. Ibrahim and P. Nath, Phys. Rev. D 88, 013019 (2013) arXiv:1306.2275 [hep-ph]].

[29] T. Ibrahim and P. Nath, Phys. Rev. D 78, 075013 (2008) arXiv:0806.3880 [hep-ph]].

[30] T. Ibrahim and P. Nath, Phys. Rev. D 81, 033007 (2010) arXiv:1001.0231 [hep-ph]].

[31] T. Ibrahim and P. Nath, Phys. Rev. D 84, 015003 (2011) arXiv:1104.3851 [hep-ph]].

[32] T. Ibrahim and P. Nath, Phys. Rev. D 82, 055001 (2010) arXiv:1007.0432 [hep-ph]].

[33] T. Ibrahim and P. Nath, Phys. Rev. D 87, 015030 (2013) arXiv:1211.0622 [hep-ph]].

[34] T. Ibrahim and P. Nath, Nucl. Phys. Proc. Suppl. 200-202, 161 (2010) arXiv:0910.1303 [hep-ph]].

[35] T. Ibrahim and P. Nath, Rev. Mod. Phys. 80, 577 (2008); arXiv:hep-ph/0210251. A. Pilaftsis, hep-ph/9908373.

[36] K. Fujikawa, B. W. Lee, and A. I. Sanda, Phys. Rev. D6, 2923 (1972); R. Jackiw and S. Weinberg, Phys. Rev. D5, 2473 (1972); G. Altarelli, N. Cabbibo, and L. Maiani, Phys. Lett. B40, 415 (1972); I. Bars and M. Yoshimura, Phys. Rev. D6, 374 (1972); W. A. Bardeen, R. Gastmans, and B. E. Lautrup, Nucl. Phys. B46, 315 (1972).

[37] S. Eidelman and M. Passera, Mod. Phys. Lett. A 22, 159 (2007) hep-ph/0701260]; S. Eidelman, M. Giacomini, F. V. Ignatov and M. Passera, Nucl. Phys. Proc. Suppl. 169, 226 (2007) hep-ph/0702026].

[38] J. Abdallah et al. [DELPHI Collaboration], Eur. Phys. J. C 35, 159 (2004) hep-ex/0406010.

[39] G. A. Gonzalez-Sprinberg, A. Santamaria and J. Vidal, Nucl. Phys. B 582 (2000) 3.

[40] A. Lusiani, Nucl. Phys. Proc. Suppl. 218 (2011) 335; T. Aushev et al., arXiv:1002.5012 [hepex]; J. Bernabeu, G. A. Gonzalez-Sprinberg, J. Papavassiliou and J. Vidal, Nucl. Phys. B 790 (2008) 160; J. Bernabeu, G. A. Gonzalez-Sprinberg and J. Vidal, JHEP 0901 (2009) 062; M. Fael, Study of the anomalous magnetic moment of the $\tau$ lepton via its radiative leptonic decays, M.Sc. Thesis (2010), University of Padua; M. L. Laursen, M. A. Samuel and A. Sen, Phys. Rev. D 29 (1984) 2652 [Erratum-ibid. D 56 (1997) 3155]; 
[41] T. Ibrahim, U. Chattopadhyay and P. Nath, Phys. Rev. D 64, 016010 (2001) hep$\mathrm{ph} / 0102324$. 